\title{
WEIGHTED LOGICS FOR NESTED WORDS AND ALGEBRAIC FORMAL POWER SERIES
}

\author{
CHRISTIAN MATHISSEN
}

Institut für Informatik, Universität Leipzig, 04009 Leipzig, Germany

e-mail address: mathissen@informatik.uni-leipzig.de

\begin{abstract}
Nested words, a model for recursive programs proposed by Alur and Madhusudan, have recently gained much interest. In this paper we introduce quantitative extensions and study nested word series which assign to nested words elements of a semiring. We show that regular nested word series coincide with series definable in weighted logics as introduced by Droste and Gastin. For this we establish a connection between nested words and the free bisemigroup. Applying our result, we obtain characterizations of algebraic formal power series in terms of weighted logics. This generalizes results of Lautemann, Schwentick and Thérien on context-free languages.
\end{abstract}

\section{INTRODUCTION}

Model checking of finite state systems has become an established method for automatic hardware and software verification and led to numerous verification programs used in industrial application. In order to verify recursive programs it is necessary to model them as pushdown systems rather than finite automata. This has motivated Alur and Madhusudan [3, 4] to define regular nested word languages and visibly pushdown languages. The latter is a proper subclass of the context-free languages and exceeds the regular languages. Both classes are closely related. Nested words on the one hand have a linear sequential structure and on the other hand have a hierarchical structure. This way they may also be used to model linguistic data as well as semistructured data such as XML documents. Nested words and visibly pushdown languages gained much interest and set a starting point for a new research field (see e.g. [1,2, 7] among many others).

The goal of this paper is: 1 . to introduce a quantitative automaton model and a quantitative logic for nested words that are equally expressive, 2. to establish a connection between nested words and alternating texts, a graph representation of the free bisemigroup which is an object studied by Ésik and Németh [17] and Hashiguchi et al. [19 21], 3. to give a characterization of the important class of algebraic formal power series by means of weighted logics.

1998 ACM Subject Classification: F.1.1, F.1.2, F.4.1, F.4.3.

Key words and phrases: nested words, algebraic formal power series, weighted automata, weighted logics. An extended abstract of this paper appeared in the proceedings of the 35th ICALP, Reykjavik, 2008 [29]. Supported by the Graduiertenkolleg 446 of the German Research Foundation (DFG). 
In order to model quantitative aspects, extensions of existing models such as weighted automata were investigated. There, transitions of automata additionally carry a weight which can be of very different nature (e.g. counting, probabilities, etc.). In fact, weighted automata have found many different applications e.g. in image processing [10, in speech recognition [34] or as a model for probabilistic systems [5, 6]. In this paper we introduce and investigate weighted nested word automata which may serve as a quantitative model for sequential programs with recursive procedure calls. Due to the fact that we define them over arbitrary semirings, they are very flexible and can model, for example, probabilistic or stochastic programs of recursive nature as well as quantitative database queries.

Since weighted nested word automata and weighted pushdown automata are closely related, one should also mention that weighted pushdown systems have been applied to data flow analysis (see e.g. [23, 24]). There, however, the emphasize lies on the (weighted) configuration graph of the system which is used to model the state space of a program. Weights are incorporated in order to model, for example, the data of the program. In [23,24] weighted versions of reachability problems in such graphs were considered.

In this paper we are interested in the semantics of a weighted automaton given as a mapping which assigns a value to each nested word. As the first main result of this paper we characterize the expressiveness of weighted nested word automata using weighted logics, generalizing a result of Alur and Madhusudan. Weighted logics were introduced by Droste and Gastin [11. They enriched the classical language of monadic second-order logic with values from a semiring in order to add quantitative expressiveness. This way one may now e.g. express how often a certain property holds, how much execution time a process needs or how reliable it is. The result of Droste and Gastin has been extended to infinite words, (infinite) trees, texts, pictures and traces [14, 15, 18, 28, 33, 36]. We note, moreover, that a restriction of Lukasiewicz multi-valued logic coincides with this weighted logics [38].

In order to prove our result mentioned above we establish a new connection between alternating texts and nested words and reduce the result to an analogous one for alternating texts. The class of alternating texts, introduced by Ehrenfeucht and Rozenberg [16], forms the free bisemigroup which was also investigated by Hashiguchi et al. [19 21]. Moreover, a language theory for series-parallel-biposets, a different representation of the free bisemigroup, was developed by Ésik and Németh [17]. Besides the author's opinion that a reduction to a previously known result is mathematically more elegant than e.g. a structural induction, the approach admits the advantage that it gives insight into relationships and similarities between different structures considered in the literature and therefore offers benefits. For example, decidability results for the emptiness and equivalence problem come almost for free as a corollary. Note that this extends the classical satisfiability problem for monadic second order logic, which is one motivation of transforming formulas in automata.

Furthermore, we can use the connection again in this paper to obtain a new characterization of algebraic formal power series. The latter form an important generalization of context-free languages. Algebraic formal power series were considered initially already by Chomsky and Schützenberger [8] and have since been intensively studied by Kuich and others. For a survey see [25] or [26]. Using projections of nested word series and applying the logical characterization of weighted nested word automata, we are able to give a characterization of algebraic formal power series in terms of weighted logics, generalizing a result of Lautemann, Schwentick and Thérien [27] on context-free languages. The connection between alternating texts and nested words is then used to also generalize a second 
characterization of [27], thereby giving a different proof also for the result of Lautemann, Schwentick and Thérien.

The paper is organized as follows. In Section 2 we introduce nested words, weighted automata for nested words and give an example for them. In Section 3 we introduce weighted logics for nested words, introduce different fragments of the latter and state the first main result, the characterization of regular nested word series in terms of weighted logics. In Section 4 we introduce alternating texts, a graph representation of the free bisemigroup and define a weighted version of Ésik and Németh's parenthesizing automata operating over elements of the free bisemigroup. Next, in Section 5, we define an embedding of nested words into alternating texts and show that we can translate weighted formulae as well as automata back and forth with respect to this embedding. This gives the proof of the first main result. After that, in Section 6, we apply the result and obtain characterizations of algebraic formal power series in terms of weighted logics.

An extended abstract of this paper appeared as [29]. This paper differs from it in the following way. First, full proofs are included. Second, the first main result, the logical characterization of regular nested word series, has been extended and it is shown that an existential fragment of weighted logics suffices to characterize weighted automata over nested words. Third, rather than translating nested words to sp-biposets, the graph representation of the free bisemigroup used by Ésik and Németh [17, we translate it to alternating texts, a different representation. This admits the advantage that we can more easily obtain a second characterization of algebraic formal power series in terms of weighted logics. This second characterization, which we include here in full length, was only sketched in the concluding remarks of [29] and gives the fourth main difference.

\section{Weighted Automata on Nested Words}

In this section we recall the notion of nested words which was introduced by Alur and Madhusudan [4] and we define weighted automata for them. Let $\Delta$ be a finite alphabet and let $\Delta^{+}$be the free semigroup of finite but non-empty words. Let $w=a_{1} \ldots a_{n} \in \Delta^{+}$. The length of $w$ is $|w|=n$. A nesting relation $\nu$ of width $n(n \in \mathbb{N})$ is a binary relation on $[n]=\{1, \ldots, n\}$ such that for all $1 \leq i, j \leq n$ :

(1) if $\nu(i, j)$, then $i<j$,

(2) if $\nu(i, j)$ and $\nu\left(i, j^{\prime}\right)$, then $j=j^{\prime}$ and if $\nu(i, j)$ and $\nu\left(i^{\prime}, j\right)$, then $i=i^{\prime}$,

(3) if $\nu(i, j)$ and $\nu\left(i^{\prime}, j^{\prime}\right)$ and $i<i^{\prime}$ then either $j<i^{\prime}$ or $j^{\prime}<j$.

If $\nu(i, j)$, we say $i$ is a call position and $j$ is a return position. Any $1 \leq i \leq n$ which is neither a call nor a return position is called an internal position. We collect all nesting relations of width $n$ in Nest $_{n}$.

Definition 2.1 (Alur \& Madhusudan [4]). A nested word (over $\Delta$ ) is a pair $(w, \nu)$ such that $w \in \Delta^{+}$and $\nu$ is a nesting relation of width $|w|$.

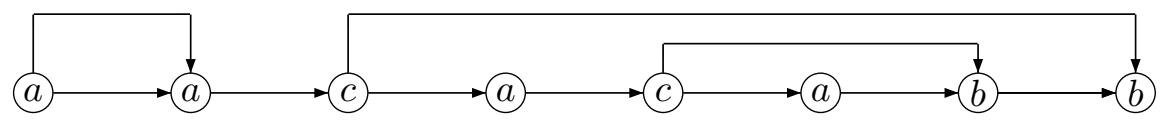

Figure 1: A visualization of the nested word (aacacabb, $\{(1,2),(3,8),(5,7)\})$ 
We collect all nested words over $\Delta$ in $\mathrm{NW}(\Delta)$. Let $n w=(w, \nu) \in \mathrm{NW}(\Delta)$ where $w=a_{1} \ldots a_{n}$. The factor $n w[i, j]$ for $1 \leq i \leq j \leq n$ is the restriction of $n w$ to the positions from $i$ to $j$; more formally $n w[i, j]=\left(a_{i} \ldots a_{j}, \nu[i, j]\right)$ where $\nu[i, j]=\{(k, \ell) \mid 1 \leq k, \ell \leq$ $j-i+1,(k+i-1, \ell+i-1) \in \nu\}$. Furthermore, we say a pair $(k, \ell) \in \nu$ is a surface arch of $n w$ if there does not exist $\left(k^{\prime}, \ell^{\prime}\right) \in \nu$ with $k^{\prime}<k<\ell<\ell^{\prime}$.

Nested words have been introduced in order to model executions of recursive programs as well as nested data structures such as XML documents. Here, we model quantitative behavior of systems or documents such as the runtime or the probability of an execution of a randomized program, or the number of occurrences of a certain type of entry in an XML document. We do this by assigning to a nested word a quantity expressing, for example, the runtime or the probability or the number of entries.

\section{Example 2.2.}

(1) As Alur and Madhusudan point out, XML documents or bibtex databases can naturally be modeled as nested words, where the nesting relation captures open and close tags [4]. Suppose we model bibtex databases as nested words. Then we may assign to a nested word e.g. the number of technical reports it stores.

(2) Probabilistic automata have been used to model systems with uncertainty, such as communication systems over lossy channels, to model fault-tolerant systems or to model randomized programs. Consider the randomized recursive pseudo-procedure bar where flip(Y) means flipping a fair coin Y. Consider furthermore the alphabet $\Delta=\{r, w, b, c a l l, r e t\}$ of atomic events which stand for read, write, beep, call and return. Now, an execution of bar could be as follows: $\operatorname{read}(\mathrm{x})$, flip a coin and see tail, call recursively bar, $\operatorname{read}(\mathrm{x})$, flip a coin and see head, beep, flip a coin and see tail, return from the re-

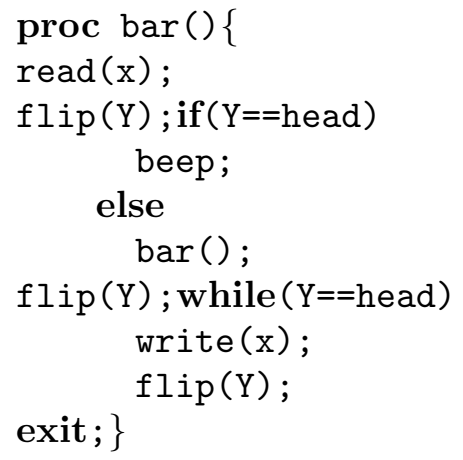
cursive call, flip a coin and see head, write(x), flip a coin and see head, write(x), flip a coin and see tail, exit the program. Then the nested word $n w=(w, \nu)$ defined by $w=$ r.call.r.b.ret.w.w.ret and $\nu=\{(2,5)\}$ models this execution of bar where $\nu$ encodes the recursive call of bar. We calculate the probability of the execution by multiplying the probability of each atomic action (probability $1 / 2$ for those actions that depend on a coin flip), i.e. $1 \cdot 1 / 2 \cdot 1 \cdot 1 / 2 \cdot 1 / 2 \cdot 1 / 2 \cdot 1 / 2 \cdot 1 / 2=1 / 64$. We will model bar using a weighted nested word automaton in Example 2.4. below.

To be as flexible as possible, we take the quantities we assign to a nested word from a commutative semiring. A commutative semiring $\mathbb{K}$ is an algebraic structure $(\mathbb{K},+, \cdot, 0,1)$ such that $(\mathbb{K},+, 0)$ and $(\mathbb{K}, \cdot, 1)$ are commutative monoids, multiplication distributes over addition and 0 is absorbing, i.e. $0 \cdot k=k \cdot 0=0$ for all $k \in \mathbb{K}$. For example the natural numbers $(\mathbb{N},+, \cdot, 0,1)$ form a commutative semiring. Other important examples are also the tropical semiring $(\mathbb{Z} \cup\{\infty\}$, min, $+, \infty, 0)$ and the arctic or max-plus semiring $(\mathbb{Z} \cup\{-\infty\}$, $\max ,+,-\infty, 0)$ which have been used to model real-time systems or discrete event systems. These semirings possess the property that any finitely generated submonoid of $(\mathbb{K},+, 0)$ is finite. Such semirings are called additively locally finite. Another important example of an additively locally finite semiring is the probabilistic semiring $([0,1], \max , \cdot, 0,1)$. We call a semiring locally finite if any finitely generated subsemiring is finite. Examples 
include any Boolean algebra such as the trivial Boolean algebra $\mathbb{B}=(\{0,1\}, \vee, \wedge, 0,1)$ as well as $\left(\mathbb{R}_{+} \cup\{\infty\}\right.$, max, $\left.\min , 0, \infty\right)$ and the fuzzy semiring $([0,1]$, $\max , \min , 0,1)$.

In the following let $\mathbb{K}$ be a commutative semiring such that $0 \neq 1$.

Definition 2.3. A weighted nested word automaton (WNWA for short) is a quadruple $\mathcal{A}=(Q, \iota, \delta, \kappa)$ where $\delta=\left(\delta_{\text {call }}, \delta_{\text {int }}, \delta_{\text {ret }}\right)$ such that

(1) $Q$ is a finite set of states,

(2) $\delta_{\text {call }}, \delta_{\text {int }}: Q \times \Delta \times Q \rightarrow \mathbb{K}$ are the call and internal transition functions,

(3) $\delta_{\text {ret }}: Q \times Q \times \Delta \times Q \rightarrow \mathbb{K}$ is the return transition function,

(4) $\iota, \kappa: Q \rightarrow \mathbb{K}$ are the initial and final distribution.

A run of $\mathcal{A}$ on $n w=\left(a_{1} \ldots a_{n}, \nu\right)$ is a sequence of states $r=\left(q_{0}, \ldots, q_{n}\right)$; we also write $r: q_{0} \stackrel{n w}{\rightarrow} q_{n}$. The weight of $r$ at position $1 \leq j \leq n$ is given by

$$
\operatorname{wgt}_{\mathcal{A}}(r, j)= \begin{cases}\delta_{\text {call }}\left(q_{j-1}, a_{j}, q_{j}\right) & \text { if } \nu(j, i) \text { for some } j<i \leq n \\ \delta_{\text {int }}\left(q_{j-1}, a_{j}, q_{j}\right) & \text { if } j \text { is an internal position } \\ \delta_{\text {ret }}\left(q_{j-1}, q_{i-1}, a_{j}, q_{j}\right) & \text { if } \nu(i, j) \text { for some } 1 \leq i<j .\end{cases}
$$

Now, the weight of $r$ is $\operatorname{wgt}_{\mathcal{A}}(r)=\prod_{1 \leq j \leq n} \operatorname{wgt}_{\mathcal{A}}(r, j)$ and the behavior $\|\mathcal{A}\|: \operatorname{NW}(\Delta) \rightarrow \mathbb{K}$ of $\mathcal{A}$ is defined by

$$
\|\mathcal{A}\|(n w)=\sum_{q_{0}, q_{n} \in Q} \iota\left(q_{0}\right) \cdot \sum_{r: q_{0} \stackrel{n w}{\rightarrow} q_{n}} \operatorname{wgt}_{\mathcal{A}}(r) \cdot \kappa\left(q_{n}\right) .
$$

A function $S: \mathrm{NW}(\Delta) \rightarrow \mathbb{K}$ is called a nested word series. As for formal power series we write $(S, n w)$ for $S(n w)$. We define the scalar multiplication . and the sum + pointwise, i.e. for $k \in \mathbb{K}$ and any two nested word series $S_{1}, S_{2}$ we let $\left(k \cdot S_{1}, n w\right)=k \cdot\left(S_{1}, n w\right)$ and $\left(S_{1}+S_{2}, n w\right)=\left(S_{1}, n w\right)+\left(S_{2}, n w\right)$ for all $n w \in \mathrm{NW}(\Delta)$. For $L \subseteq \mathrm{NW}(\Delta)$ let $\mathbb{1}_{L}$ be the characteristic series of $L$, i.e. the series that assumes 1 for all $n w \in L$ and 0 otherwise. A nested word series $S$ is regular if there is a WNWA $\mathcal{A}$ such that $\|\mathcal{A}\|=S$. For $\mathbb{K}=\mathbb{B}$, i.e. when $\delta_{\text {call }}, \delta_{\text {int }}$ and $\delta_{\text {ret }}$ are subsets of $Q \times \Delta \times Q$ and $Q \times Q \times \Delta \times Q$, or in other words when the transitions do not carry a weight, Definition 2.3 is equivalent to the definition of a (unweighted) nested word automaton [4]. A language of nested words $L \subseteq \mathrm{NW}(\Delta)$ is then called regular if it is accepted by a nested word automaton. It is easy to see that this is the case iff the characteristic series $\mathbb{1}_{L}: \mathrm{NW}(\Delta) \rightarrow \mathbb{B}$ is regular.

Example 2.4. The procedure bar of Example 2.2 can be modeled by a WNWA over $\mathbb{K}=([0,1], \max , \cdot, 0,1)$ with four states $\left\{q_{1}, \ldots, q_{4}\right\}$. The transitions (only those with nonzero weight) are given as follows. We let $\iota\left(q_{1}\right)=1$ and $\kappa\left(q_{4}\right)=1$. Moreover,

$$
\begin{array}{lc}
\delta_{\text {int }}\left(q_{1}, r, q_{2}\right)=1, & \delta_{\text {int }}\left(q_{2}, b, q_{3}\right)=\delta_{\text {int }}\left(q_{3}, w, q_{3}\right)=\delta_{\text {int }}\left(q_{3}, \text { ret }, q_{4}\right)=1 / 2 \\
\delta_{\text {call }}\left(q_{2}, \text { call }, q_{1}\right)=1 / 2, & \delta_{\text {ret }}\left(q_{3}, q_{2}, \text { ret }, q_{3}\right)=1 / 2 .
\end{array}
$$

Intuitively, each of the states corresponds to a line in the procedure bar which is the next to be executed. $q_{1}$ corresponds to line $2, q_{2}$ corresponds to line $3, q_{3}$ corresponds to line 7 and $q_{4}$ is only reached at the end of an execution. Consider the nested word $n w$ of Example 2.2(2). There is exactly one run $r: q_{1} \stackrel{n w}{\rightarrow} q_{4}$ with $\operatorname{wgt}(r) \neq 0$. We start in state $q_{1}$ execute $r$ and change to $q_{2}$. We then call and change back to $q_{1}$. After that we execute $r$ again and change to state $q_{2}$. We then execute $b$ and change to $q_{3}$. We return and stay in $q_{3}$. Now we execute $w$ twice while staying in $q_{3}$ and finally end at state $q_{4}$. Observe that the automaton assigns $1 / 64$ to the nested word $n w$. 


\section{Weighted Logics}

In this section we introduce another formalism for specifying nested word series. For this we interpret a nested word $n w=\left(a_{1} \ldots a_{n}, \nu\right)$ as a relational structure consisting of the domain $\operatorname{dom}(n w)=[n]$ together with the unary relations $\operatorname{Lab}_{a}=\left\{i \in \operatorname{dom}(n w) \mid a_{i}=a\right\}$ for all $a \in \Delta$, the binary relation $\nu$ and the usual $\leq$ relation on $\operatorname{dom}(n w)$.

First, we recall classical monadic second-order logic. The set $\operatorname{MSO}(\Delta, \leq, \nu)$ (we also write MSO for short) is given by the following grammar.

$$
\varphi \quad::=\quad x=y\left|\operatorname{Lab}_{a}(x)\right| x \leq y|\nu(x, y)| x \in X|\varphi \vee \varphi| \neg \varphi|\exists x . \varphi| \exists X . \varphi
$$

where $a$ ranges over $\Delta$, where $x, y$ are first-order variables and where $X$ is a second-order variable. As usual we abbreviate $x<y=\neg(y \leq x), \varphi \rightarrow \psi=\neg \varphi \vee \psi$ and $\varphi \leftrightarrow \psi=$ $(\varphi \rightarrow \psi) \wedge(\psi \rightarrow \varphi)$ for any $\varphi, \psi \in \mathrm{MSO}$.

Let $\varphi \in \mathrm{MSO}$ and let $\operatorname{Free}(\varphi)$ denote the set of variables that occur free in $\varphi$. Let $\mathcal{V}$ be a finite set of first-order and second-order variables such that $\operatorname{Free}(\varphi) \subseteq \mathcal{V}$. A $(\mathcal{V}, n w)$ assignment $\gamma$ is a mapping from $\mathcal{V}$ to the powerset $\mathscr{P}(\operatorname{dom}(n w))$ such that first-order variables are mapped to singletons. For $i \in \operatorname{dom}(n w)$ and $T \subseteq \operatorname{dom}(n w)$ we denote by $\gamma[x \rightarrow i]$ (resp. $\gamma[X \rightarrow T])$ the $(\mathcal{V} \cup\{x\}, n w)$-assignment (resp. $(\mathcal{V} \cup\{X\}, n w)$-assignment) which equals $\gamma$ on $\mathcal{V} \backslash\{x\}$ (resp. $\mathcal{V} \backslash\{X\}$ ) and assumes $\{i\}$ for $x$ (resp. $T$ for $X$ ). We write $(n w, \gamma) \models \varphi$ if $\varphi$ holds in $n w$ under the assignment $\gamma$. We write $\varphi\left(x_{1}, \ldots, x_{n}, X_{1}, \ldots, X_{m}\right)$ if $\operatorname{Free}(\varphi) \subseteq\left\{x_{1}, \ldots, x_{n}, X_{1}, \ldots, X_{m}\right\}$. In this case write $n w \models \varphi\left[i_{1}, \ldots, i_{n}, T_{1}, \ldots, T_{m}\right]$ whenever we have $(n w, \gamma) \models \varphi$ if $\gamma\left(x_{j}\right)=\left\{i_{j}\right\}$ and $\gamma\left(X_{j}\right)=T_{j}$. This is justified by the fact that $(n w, \gamma) \models \varphi$ only depends on the restriction $\gamma_{\mid \operatorname{Free}(\varphi)}$ of $\gamma$ to $\operatorname{Free}(\varphi)$. Let $\mathscr{L} \mathcal{V}(\varphi)=\{(n w, \gamma) \mid n w \in \mathrm{NW}(\Delta), \gamma$ is a $(\mathcal{V}, n w)$-assignment, $(n w, \gamma) \models \varphi\}$. Abbreviate $\mathscr{L}(\varphi)=\mathscr{L}_{\text {Free }(\varphi)}(\varphi)$. Note that in case that $\varphi$ is a sentence, i.e. Free $(\varphi)=\emptyset$, we consider $\mathscr{L}(\varphi)$ as a subset of $\mathrm{NW}(\Delta)$.

Let $Z \subseteq$ MSO. A language $L \subseteq \mathrm{NW}(\Delta)$ is $Z$-definable if $L=\mathscr{L}(\varphi)$ for a sentence $\varphi \in Z$. Formulae containing no quantification at all are called propositional. First-order formulae, i.e. formulae containing only quantification over first-order variables are collected in FO. The class EMSO consists of all formulae $\varphi$ of the form $\exists X_{1} \ldots \exists X_{m}$. $\psi$ where $\psi \in \mathrm{FO}$. Alur and Madhusudan showed that monadic second-order logic and nested word automata are equally expressive.

Theorem 3.1 (Alur \& Madhusudan [3,4]). A nested word language $L \subseteq \mathrm{NW}(\Delta)$ is regular iff $L$ is MSO-definable iff $L$ is EMSO-definable.

We now turn to weighted monadic second-order logic as introduced in [11. The set $\operatorname{MSO}(\mathbb{K}, \Delta, \leq, \nu)$ (once again we shortly write $\mathrm{MSO}(\mathbb{K})$ ) of weighted MSO formulae over $\mathbb{K}$ is given by the following grammar:

$$
\begin{aligned}
& \varphi \quad::=\quad k|x=y| \operatorname{Lab}_{a}(x)|x \leq y| \nu(x, y) \mid x \in X \\
&|\neg(x=y)| \neg \operatorname{Lab}_{a}(x)|\neg x \leq y| \neg \nu(x, y) \mid \neg(x \in X) \\
&|\varphi \vee \varphi| \varphi \wedge \varphi|\exists x . \varphi| \exists X . \varphi|\forall x . \varphi| \forall X . \varphi
\end{aligned}
$$

where $k \in \mathbb{K}$, where $a$ ranges over $\Delta$, where $x, y$ are first-order variables and where $X$ is a second-order variable. Note that we allow negation only for atomic formulae, i.e. for the formulae $x=y, \operatorname{Lab}_{a}(x), x \leq y, \nu(x, y)$ and $x \in X$. This is because in general semirings we do not have a natural complement and hence it is not clear how to define the semantics of negation for values other than 0 and 1 (cf. [11]). 
Let $\varphi \in \operatorname{MSO}(\mathbb{K})$ and $\operatorname{Free}(\varphi) \subseteq \mathcal{V}$. The weighted semantics $\llbracket \varphi \rrbracket \mathcal{V}$ of $\varphi$ is a function assigning a value in $\mathbb{K}$ to a nested word $n w$ and a $(\mathcal{V}, n w)$-assignment $\gamma$. To each such pair $(n w, \gamma)$ we assign an element of $\mathbb{K}$ inductively as follows. For $k \in \mathbb{K}$ we put $\llbracket k \rrbracket \mathcal{V}(n w, \gamma)=k$. For every other atomic formula or negated atomic formula $\varphi$ the semantics $\llbracket \varphi \rrbracket \mathcal{V}$ is given by the characteristic function $\mathbb{1}_{\mathscr{L}_{\mathcal{V}}(\varphi)}$. Moreover, we define

$$
\begin{array}{lll}
\llbracket \varphi \vee \psi \rrbracket \mathcal{V}(n w, \gamma) & = & \llbracket \varphi \rrbracket \mathcal{V}(n w, \gamma)+\llbracket \psi \rrbracket \mathcal{V}(n w, \gamma), \\
\llbracket \varphi \wedge \psi \rrbracket \mathcal{V}(n w, \gamma) & = & \llbracket \varphi \rrbracket \mathcal{V}(n w, \gamma) \cdot \llbracket \psi \rrbracket \mathcal{V}(n w, \gamma), \\
\llbracket \exists x . \varphi \rrbracket \mathcal{V}(n w, \gamma) & = & \sum_{i \in \operatorname{dom}(n w)} \llbracket \varphi \rrbracket \mathcal{V} \cup\{x\} \\
\llbracket \exists X . \varphi \rrbracket \mathcal{V}(n w, \gamma) & = & \sum_{T \subseteq \operatorname{dom}(n w)} \llbracket \varphi \rrbracket \mathcal{V} \cup\{X\} \\
\llbracket \forall x . \varphi \rrbracket \mathcal{V}(n w, \gamma) & = & \prod_{i \in \operatorname{dom}(n w)} \llbracket \varphi \rrbracket \rrbracket_{\mathcal{V} \cup\{x\}}(n w, \gamma[X \rightarrow i]), \\
\llbracket \forall X . \varphi \rrbracket \mathcal{V}(n w, \gamma) & = & \prod_{T \subseteq \operatorname{dom}(n w)} \llbracket \varphi \rrbracket \rrbracket_{\mathcal{V} \cup\{X\}}(n w, \gamma[X \rightarrow T]),
\end{array}
$$

We put $\llbracket \varphi \rrbracket=\llbracket \varphi \rrbracket_{\operatorname{Free}(\varphi)}$. Observe that in the case where $\varphi$ is a sentence, $\llbracket \varphi \rrbracket$ can be considered as a series from $\mathrm{NW}(\Delta)$ to $\mathbb{K}$.

Remark 3.2. A formula $\varphi \in \mathrm{MSO}(\mathbb{K})$ which does not contain a subformula $k \in \mathbb{K}$ can be interpreted as an unweighted formula. We will use this implicitly in the sequel. Moreover, note that if $\mathbb{K}$ is the Boolean semiring $\mathbb{B}$, then weighted logics and classical MSO logic coincide. In this case $k$ is either 0 (false) or 1 (true).

\section{Example 3.3.}

(1) As in Example 2.2 suppose we model bibtex databases as nested words. Moreover, assume that tecrep $\in \Delta$ marks the beginning of an entry containing a technical report. Now, let $\mathbb{K}=\mathbb{N}$ be the semiring of the natural numbers. Then $(\llbracket \exists x$. Lab tecrep $(x) \rrbracket, n w)$ counts the number of technical reports of the bibtex database modeled by $n w$.

(2) Again let $\mathbb{K}=\mathbb{N}$. Consider the formula $\varphi=\forall x . \exists y .1$. Then $\left(\llbracket \exists x .1 \rrbracket,\left(a_{1} \ldots a_{n}, \nu\right)\right)=n$ and $\left(\llbracket \forall y \cdot \exists x .1 \rrbracket,\left(a_{1} \ldots a_{n}, \nu\right)\right)=n^{n}$. It can be shown as for words that $\llbracket \varphi \rrbracket$ is not regular as it grows too fast (cf. Example 3.4 in [1] ).

Let $Z \subseteq \mathrm{MSO}(\mathbb{K})$. A series $S: \mathrm{NW}(\Delta) \rightarrow \mathbb{K}$ is $Z$-definable if $S=\llbracket \varphi \rrbracket$ for a sentence $\varphi \in Z$. Example 3.3(2) shows that unrestricted application of universal quantification does not preserve regularity. Therefore we now define different fragments of $\mathrm{MSO}(\mathbb{K})$.

Note that the fragment RMSO $(\mathbb{K})$, the collection of restricted formulae, which was considered in [11] and which characterizes regular formal power series is a semantic restriction, and it is not clear whether membership in $\operatorname{RMSO}(\mathbb{K})$ can be decided. In order to have a decidable fragment, we now syntactically define the fragment $\operatorname{sRMSO}(\mathbb{K})$. For this we follow the approach of [12].

The idea is to restrict universal first-order quantification to formulae having a semantics that takes on only finitely many values. To this aim we start by identifying a class of formulae $\varphi$ that take on values 0 and 1 only, more precisely we will have $\mathbb{1}_{\mathscr{L}_{\mathcal{V}}(\varphi)}=\llbracket \varphi \rrbracket \mathcal{V}$. The problem that arises is that by definition of the semantics, $\vee$ gets translated by means of + . Hence, for a formula $\varphi=\varphi_{1} \vee \varphi_{2}$ we only want to evaluate $\varphi_{2}$ if $\varphi_{1}$ evaluates to 0 , otherwise we might end up with a sum greater than one. A similar problem occurs for $\exists x$. and $\exists X$. 
Given a classical (unweighted) MSO-formula $\varphi$ we assign to it formulae $\varphi^{+}$and $\varphi^{-}$such that $\llbracket \varphi^{+} \rrbracket=\mathbb{1}_{\mathscr{L}(\varphi)}$ and $\llbracket \varphi^{-} \rrbracket=\mathbb{1}_{\mathscr{L}(\neg \varphi)}$. The crucial point is that we have a linear order at disposal.

(1) If $\varphi$ is of the form $x=y, \operatorname{Lab}_{a}(x), x \leq y, \nu(x, y), x \in X$ then $\varphi^{+}=\varphi$ and $\varphi^{-}=\neg \varphi$.

(2) If $\varphi=\neg \psi$, then $\varphi^{+}=\psi^{-}$and $\varphi^{-}=\psi^{+}$.

(3) If $\varphi=\psi \vee \psi^{\prime}$, then $\varphi^{+}=\psi^{+} \vee\left(\psi^{-} \wedge \psi^{\prime+}\right)$ and $\varphi^{-}=\psi^{-} \wedge \psi^{\prime-}$.

(4) If $\varphi=\exists x . \psi(x)$, then $\varphi^{+}=\exists x . \psi(x)^{+} \wedge \forall y .(y<x \wedge \psi(y))^{-}$and $\varphi^{-}=\forall x . \psi(x)^{-}$.

In order to disambiguate set quantification, we have to define a linear order on the subsets of the domain of a nested word or equivalently on nested words (of fixed length) over the alphabet $\{0,1\}$. We take the lexicographic order $<$ which is given by the following formula.

$$
X<Y=\exists y . y \in Y \wedge \neg y \in X \wedge \forall z \cdot[z<y \rightarrow(z \in X \leftrightarrow z \in Y)]^{+}
$$

Now we proceed:

(5) If $\varphi=\exists X . \psi(X)$, then $\varphi^{+}=\exists X . \psi(X)^{+} \wedge \forall Y .(Y<X \wedge \psi(Y))^{-}$and $\varphi^{-}=\forall X . \psi(X)^{-}$. Formulae of the form $\varphi^{+}$or $\varphi^{-}$for some $\varphi \in$ MSO are called syntactically unambiguous. Observe, if $\varphi$ is syntactically unambiguous, then $\llbracket \varphi \rrbracket \mathcal{V}=\mathbb{1}_{\mathscr{L}_{\mathcal{V}}(\varphi)}$ for any finite set of variables $\mathcal{V} \supseteq \operatorname{Free}(\varphi)$. In the following, we shortly write $\varphi \stackrel{+}{\rightarrow} \psi$ for $\varphi^{-} \vee\left(\varphi^{+} \wedge \psi\right)$ for any two weighted formulae $\varphi, \psi$ where $\varphi$ does not contain subformulae of the form $k(k \in \mathbb{K})$ and hence is also a classical formula.

We define aUMSO $(\mathbb{K})$, the collection of almost unambiguous formulae, to be the smallest subset of $\mathrm{MSO}(\mathbb{K})$ containing all constants $k(k \in \mathbb{K})$ and all syntactically unambiguous formulae which is closed under conjunction and disjunction. Using the distributivity, observe that for any $\psi \in \operatorname{aUMSO}(\mathbb{K})$ there is a formula $\psi^{\prime}$ of the form $\psi^{\prime}=\bigvee_{i=1}^{n}\left(k_{i} \wedge \psi_{i}\right)$ for some $k_{i} \in \mathbb{K}$ and syntactically unambiguous $\psi_{i}$ such that $\llbracket \psi \rrbracket=\llbracket \psi^{\prime} \rrbracket$ (cf. [12]). We are now ready to define the fragment $\operatorname{sRMSO}(\mathbb{K})$.

Definition 3.4. A weighted formula $\varphi$ is in $\operatorname{sRMSO}(\mathbb{K})$ (syntactically restricted MSO) if for every subformula $\vartheta$ of $\varphi$ the following two conditions hold:

(1) If $\vartheta=\forall X . \psi$ for some $\psi \in \mathrm{MSO}(\mathbb{K})$, then $\psi$ is syntactically unambiguous.

(2) If $\vartheta=\forall x \cdot \psi$ for some $\psi \in \operatorname{MSO}(\mathbb{K})$, then $\psi \in \operatorname{aUMSO}(\mathbb{K})$.

We collect in $\operatorname{sRFO}(\mathbb{K})$ all $\varphi \in \operatorname{sRMSO}(\mathbb{K})$ which do not contain any set quantification and we collect in $\operatorname{sREMSO}(\mathbb{K})$ all $\varphi \in \operatorname{sRMSO}(\mathbb{K})$ of the form $\exists X_{1} \ldots \exists X_{m} . \psi$ with $\psi \in \operatorname{sRFO}(\mathbb{K})$.

Let now $\mathrm{wUMSO}(\mathbb{K})$, the collection of weakly unambiguous formulae, be the smallest subset of $\mathrm{MSO}(\mathbb{K})$ containing all constants $k(k \in \mathbb{K})$ and all syntactically unambiguous formulae which is closed under conjunction, disjunction and existential quantification (both first- and second-order). We define the fragment $\operatorname{swRMSO}(\mathbb{K})$.

Definition 3.5. A weighted formula $\varphi$ is in $\operatorname{swRMSO}(\mathbb{K})$ (syntactically weakly restricted MSO) if for every subformula $\vartheta$ of $\varphi$ the following two conditions hold:

(1) If $\vartheta=\forall X$. $\psi$ for some $\psi \in \operatorname{MSO}(\mathbb{K})$, then $\psi$ is syntactically unambiguous.

(2) If $\vartheta=\forall x . \psi$ for some $\psi \in \operatorname{MSO}(\mathbb{K})$, then $\psi \in \mathrm{wUMSO}(\mathbb{K})$.

Clearly, aUMSO $(\mathbb{K}) \subset$ wUMSO $(\mathbb{K}) \subset \operatorname{sRMSO}(\mathbb{K}) \subset \operatorname{swRMSO}(\mathbb{K}) \subset \operatorname{MSO}(\mathbb{K})$. The first main result of this paper is the characterization of regular nested word series using weighted logics. It reads as follows. 
Theorem 3.6. Let $\mathbb{K}$ be a commutative semiring and let $S: \mathrm{NW}(\Delta) \rightarrow \mathbb{K}$ be a nested word series. Then the following holds.

(a) $S$ is regular iff it is $\mathrm{SRMSO}(\mathbb{K})$-definable iff it is $\mathrm{SREMSO}(\mathbb{K})$-definable.

(b) If $\mathbb{K}$ is additively locally finite, then $S$ is regular iff it is $\mathrm{swRMSO}(\mathbb{K})$-definable.

(c) If $\mathbb{K}$ is locally finite, then $S$ is regular iff it is $\mathrm{MSO}(\mathbb{K})$-definable.

We prove the result at the end of Section 5 by interpreting nested words in alternating texts. In the next section we introduce alternating texts and weighted automata for them.

Example 3.7. The nesting depth of a position $i$ of a nested word $n w$ is the number of open call positions (i.e. where the corresponding return position has not occurred yet including the position itself). The nesting depth of a nested word is the maximum nesting depth of its positions. Let $\mathbb{K}=(\mathbb{Z} \cup\{-\infty\}$, $\max ,+,-\infty, 0)$.

$$
\begin{aligned}
& \operatorname{open}(x)=\forall y \cdot(y \leq x \wedge \operatorname{call}(y)) \stackrel{+}{\rightarrow} 1 \wedge(y \leq x \wedge \operatorname{return}(y)) \stackrel{+}{\rightarrow}-1 \text { where } \\
& \operatorname{call}(x)=\exists y . \nu(x, y) \text { and } \operatorname{return}(x)=\exists y \cdot \nu(y, x)
\end{aligned}
$$

Then $\llbracket \exists x$.open $(x) \rrbracket$ assigns to a nested word its nesting depth. Hence, since $\exists x$.open $(x) \in$ $\operatorname{sRMSO}(\mathbb{K})$, the series is regular by Theorem 3.6 .

\section{Alternating Texts}

A bisemigroup is a set together with two associative operations. Several authors investigated the free bisemigroup as a fundamental, two-dimensional extension of classical automaton theory, see e.g. Ésik and Németh [17] and Hashiguchi et al. (e.g. 19 21]). Ésik and Németh considered as a representation for the free bisemigroup the so-called sp-biposets, a certain class of biposets. A different representation of the free bisemigroup over some finite set $\Delta$ are the so-called alternating texts [16,22]. A text over $\Delta$ is a tuple $\left(V, \lambda, \leq_{1}, \leq_{2}\right)$ where $\leq_{1}$ and $\leq_{2}$ are linear orders over a finite but non-empty domain $V$ and $\lambda: V \rightarrow \Delta$ is a labeling function. Of course we consider texts only up to isomorphism. Therefore, unless otherwise specified, the domain of a text will be $[n]=\{1, \ldots, n\}$ for some $n \in \mathbb{N}$ and $\leq_{1}$ will correspond to the canonical order on $[n]$.

We define the binary operations $\circ$ and $\bullet$, called the horizontal and vertical product, on texts as follows: Let $\tau=\left(V, \lambda, \leq_{1}, \leq_{2}\right)$ and $\tau^{\prime}=\left(V^{\prime}, \lambda^{\prime}, \leq_{1}^{\prime}, \leq_{2}^{\prime}\right)$ be two texts where we assume that $V$ and $V^{\prime}$ are disjoint. Then

$$
\begin{aligned}
& \tau_{1} \circ \tau_{2}=\left(V \uplus V^{\prime}, \lambda \cup \lambda^{\prime}, \leq_{1} \cup \leq_{1}^{\prime} \cup V \times V^{\prime}, \leq_{2} \cup \leq_{2}^{\prime} \cup V \times V^{\prime}\right), \\
& \tau_{1} \bullet \tau_{2}=\left(V \uplus V^{\prime}, \lambda \cup \lambda^{\prime}, \leq_{1} \cup \leq_{1}^{\prime} \cup V \times V^{\prime}, \leq_{2} \cup \leq_{2}^{\prime} \cup V^{\prime} \times V\right) .
\end{aligned}
$$

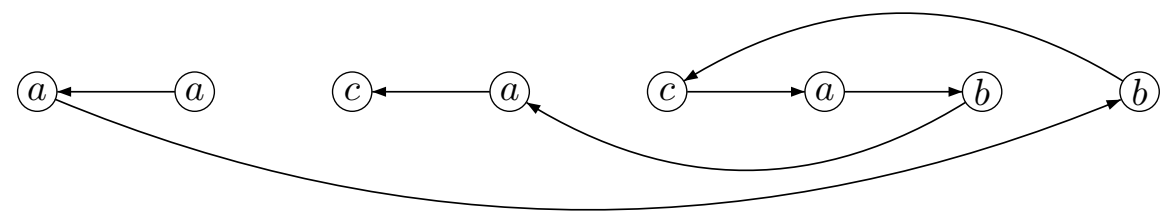

Figure 2: A visualization of the alternating text given by $(a \bullet a) \circ(c \bullet a \bullet(c \circ a \circ b) \bullet b)$. Here we only give the successor relation of the second order. The first order is given simply from the left to the right. 
Let $\operatorname{TXT}(\Delta)$ be the class of texts which can be obtained from the singleton texts by finite applications of $\circ, \bullet$. This class was named the class of alternating texts in [16]. The class $\operatorname{TXT}(\Delta)$ together with the operations $\circ, \bullet$ is the free bisemigroup over $\Delta[22]$. Let monadic second-order logic $\operatorname{MSO}\left(\Delta, \leq_{1}, \leq_{2}\right)$ and weighted logics for texts, denoted $\operatorname{MSO}\left(\mathbb{K}, \Delta, \leq_{1}, \leq_{2}\right)$ be defined along the same lines as for nested words. Moreover, define $\operatorname{sRMSO}\left(\mathbb{K}, \Delta, \leq_{1}, \leq_{2}\right)$ and $\operatorname{swRMSO}\left(\mathbb{K}, \Delta, \leq_{1}, \leq_{2}\right)$ using the linear order $\leq_{1}$.

Now we introduce weighted parenthesizing automata (cf. [28]) operating on the free bisemigroup generalizing parenthesizing automata as introduced by Ésik and Németh [17].

Definition 4.1. A tuple $\mathcal{A}=\left(\mathcal{H}, \mathcal{V}, \Omega, \mu, \mu_{\mathrm{op}}, \mu_{\mathrm{cl}}, \lambda, \gamma\right)$ is a weighted parenthesizing automaton (WPA) provided that

- $\mathcal{H}$ and $\mathcal{V}$ are finite, disjoint sets of horizontal and vertical states, respectively,

- $\Omega$ is a finite set of parentheses, 1

- $\mu:(\mathcal{H} \times \Delta \times \mathcal{H}) \cup(\mathcal{V} \times \Delta \times \mathcal{V}) \rightarrow \mathbb{K}$ is the transition function,

- $\mu_{\mathrm{op}}, \mu_{\mathrm{cl}}:(\mathcal{H} \times \Omega \times \mathcal{V}) \cup(\mathcal{V} \times \Omega \times \mathcal{H}) \rightarrow \mathbb{K}$ are the opening and closing parenthesizing functions, respectively,

- $\lambda, \gamma: \mathcal{H} \cup \mathcal{V} \rightarrow \mathbb{K}$ are the initial and final weight functions, respectively.

We now come to the notion of a run $r$ of $\mathcal{A}$. We given an inductive definition where we also define its label $\operatorname{lab}(r) \in \operatorname{TXT}(\Delta)$, its weight $\operatorname{wgt}_{\mathcal{A}}(r) \in \mathbb{K}$, its initial state $\operatorname{init}(r) \in \mathcal{H} \cup \mathcal{V}$ and its final state $\operatorname{fin}(r) \in \mathcal{H} \cup \mathcal{V}$. Formally the set of runs of $\mathcal{A}$ is the smallest set of words over the alphabet $\Delta \cup \Omega \cup \mathcal{H} \cup \mathcal{V} \cup\{(),\} \cup\{$,$\} such that:$

(1) The word $\left(q_{1}, a, q_{2}\right)$ is a run for all $\left(q_{1}, q_{2}\right) \in(\mathcal{H} \times \mathcal{H}) \cup(\mathcal{V} \times \mathcal{V})$ and $a \in \Delta$. We set

$$
\begin{aligned}
& \operatorname{lab}\left(\left(q_{1}, a, q_{2}\right)\right)=a \in \operatorname{TXT}(\Delta), \quad \operatorname{wgt}_{\mathcal{A}}\left(\left(q_{1}, a, q_{2}\right)\right)=\mu\left(q_{1}, a, q_{2}\right), \\
& \operatorname{init}\left(\left(q_{1}, a, q_{2}\right)\right)=q_{1} \text { and } \operatorname{fin}\left(\left(q_{1}, a, q_{2}\right)\right)=q_{2} .
\end{aligned}
$$

(2) If $r_{1}$ and $r_{2}$ are runs such that $\operatorname{fin}\left(r_{1}\right)=\operatorname{init}\left(r_{2}\right) \in \mathcal{H}$ (respectively such that $\operatorname{fin}\left(r_{1}\right)=$ $\left.\operatorname{init}\left(r_{2}\right) \in \mathcal{V}\right)$, then $r=r_{1} r_{2}$ is a run having

$$
\begin{array}{lr}
\operatorname{lab}(r)=\operatorname{lab}\left(r_{1}\right) \circ \operatorname{lab}\left(r_{2}\right), & \left(\operatorname{resp} . \operatorname{lab}(r)=\operatorname{lab}\left(r_{1}\right) \bullet \operatorname{lab}\left(r_{2}\right)\right), \\
\operatorname{wgt}_{\mathcal{A}}(r)=\operatorname{wgt}_{\mathcal{A}}\left(r_{1}\right) \cdot \operatorname{wgt}_{\mathcal{A}}\left(r_{2}\right), & \operatorname{init}(r)=\operatorname{init}\left(r_{1}\right) \text { and } \operatorname{fin}(r)=\operatorname{fin}\left(r_{2}\right) .
\end{array}
$$

(3) If a run $r$ resulting from 2 has $\operatorname{init}(r) \in \mathcal{H}$ (resp. $\operatorname{init}(r) \in \mathcal{V})$ and if $q_{1}, q_{2} \in \mathcal{V}$ (resp. if $\left.q_{1}, q_{2} \in \mathcal{H}\right)$ and $s \in \Omega$, then $r^{\prime}=\left(q_{1},\left({ }_{s}, \operatorname{init}(r)\right) r(\operatorname{fin}(r),)_{s}, q_{2}\right)$ is a run. We set

$$
\begin{aligned}
& \operatorname{lab}\left(r^{\prime}\right)=\operatorname{lab}(r), \quad \operatorname{init}\left(r^{\prime}\right)=q_{1} \text { and } \operatorname{fin}\left(r^{\prime}\right)=q_{2}, \\
& \operatorname{wgt}_{\mathcal{A}}\left(r^{\prime}\right)=\mu_{\mathrm{op}}\left(\left(q_{1},(s, \operatorname{init}(r))\right) \cdot \operatorname{wgt}_{\mathcal{A}}(r) \cdot \mu_{\mathrm{cl}}\left((\operatorname{fin}(r),)_{s}, q_{2}\right)\right) .
\end{aligned}
$$

Let $\tau \in \operatorname{TXT}(\Delta)$. Since in (3) above we require that the run $r$ we start with results from (2), we do not allow repeated application of (3) and therefore there are only finitely many runs $r$ of $\mathcal{A}$ with label $\tau$. Intuitively, we do not allow for doubled parentheses. If $r$ is a run of $\mathcal{A}$ with $\operatorname{lab}(r)=\tau$, $\operatorname{init}(r)=q_{1}$, fin $(r)=q_{2}$, we write $r: q_{1} \stackrel{\tau}{\rightarrow} q_{2}$. The behavior of $\mathcal{A}$ is a text series $\|\mathcal{A}\|: \operatorname{TXT}(\Delta) \rightarrow \mathbb{K}$. It is given by

$$
(\|\mathcal{A}\|, \tau)=\sum_{q_{1}, q_{2} \in \mathcal{H} \cup \mathcal{V}} \lambda\left(q_{1}\right) \cdot \sum_{r: q_{1} \rightarrow q_{2}} \operatorname{wgt}_{\mathcal{A}}(r) \cdot \gamma\left(q_{2}\right) .
$$

A text series $S$ is regular if there is a WPA $\mathcal{A}$ such that $\|\mathcal{A}\|=S$.

1 We let $s \in \Omega$ represent both an opening and a closing parentheses. To help the intuition we also write $(s \text { or })_{s}$ for $s$. 
Theorem 4.2 (see [30]). Let $\mathbb{K}$ be a commutative semiring and let $S: \operatorname{TXT}(\Delta) \rightarrow \mathbb{K}$ be an alternating text series. Then the following holds.

(a) $S$ is regular iff it is $\mathrm{SRMSO}(\mathbb{K})$-definable iff it is $\mathrm{sREMSO}(\mathbb{K})$-definable.

(b) If $\mathbb{K}$ is additively locally finite, then $S$ is regular iff it is $\mathrm{SwRMSO}(\mathbb{K})$-definable.

(c) If $\mathbb{K}$ is locally finite, then $S$ is regular iff it is $\mathrm{MSO}(\mathbb{K})$-definable.

We note that the proof in [30] is effective, i.e. given an $\operatorname{sRMSO}(\mathbb{K})(\operatorname{resp} . \operatorname{swRMSO}(\mathbb{K})$, resp. $\operatorname{MSO}(\mathbb{K}))$ formula $\varphi$ we can effectively construct a WPA $\mathcal{A}$ such that $\llbracket \varphi \rrbracket=\|\mathcal{A}\|$, and conversely, given a WPA $\mathcal{A}$ we can effectively construct $\varphi \in \operatorname{sREMSO}(\mathbb{K})$ such that $\llbracket \varphi \rrbracket=\|\mathcal{A}\|$.

\section{Interpreting Nested Words in Alternating Texts}

We will now derive similar results for nested words as for alternating texts by interpreting the different structures within each other. For this we utilize definable transductions as introduced by Courcelle [9. We only have to ensure that they preserve definability, now with respect to weighted logics. First, we introduce the notion of definable transductions. For this let $\sigma_{1}$ and $\sigma_{2}=\left(\left(R_{i}\right)_{i \in I}, \rho\right)$ be two relational signatures where $\rho: I \rightarrow \mathbb{N}_{+}$assigns to each relation symbol $R_{i}$ a positive arity. Moreover, let $\mathcal{C}_{1}$ and $\mathcal{C}_{2}$ be classes of finite $\sigma_{1^{-}}$ and $\sigma_{2}$-structures, respectively. Let monadic second-order logic $\operatorname{MSO}\left(\sigma_{1}\right)$ and $\operatorname{MSO}\left(\sigma_{2}\right)$ be defined along the lines as for nested words.

By a $\left(\sigma_{1}, \sigma_{2}\right)$-1-copying definition scheme with parameters $X_{1}, \ldots, X_{n}$ we mean a tuple $\mathcal{D}=\left(\vartheta, \delta,\left(\varphi_{i}\right)_{i \in I}\right)$ of formulae in $\operatorname{MSO}\left(\sigma_{1}\right)$ such that $\operatorname{Free}(\vartheta) \subseteq\left\{X_{1}, \ldots, X_{n}\right\}$, Free $(\delta) \subseteq$ $\left\{x_{1}, X_{1}, \ldots, X_{n}\right\}$ and Free $\left(\varphi_{i}\right) \subseteq\left\{x_{1}, \ldots, x_{\rho(i)}, X_{1}, \ldots X_{n}\right\}$ for all $i \in I$.

Let $\mathcal{D}$ be a $\left(\sigma_{1}, \sigma_{2}\right)$-1-copying definition scheme, let $s_{1} \in \mathcal{C}_{1}$ and let $T_{1}, \ldots, T_{n}$ subsets of the domain $\operatorname{dom}\left(s_{1}\right)$ of $s_{1}$ such that $s_{1} \models \vartheta\left[T_{1}, \ldots, T_{n}\right]$. Then define the $\sigma$-structure $\operatorname{def}_{\mathcal{D}}\left(s_{1}, T_{1}, \ldots, T_{n}\right)=s_{2}$ with domain $\operatorname{dom}\left(s_{2}\right) \subseteq \operatorname{dom}\left(s_{1}\right)$ and interpretations of relation symbols $R_{i}^{s_{2}}$ given as follows:

$$
\begin{aligned}
v \in \operatorname{dom}\left(s_{2}\right) \Leftrightarrow s_{1} \models \delta\left[v, T_{1}, \ldots, T_{n}\right] \text { for all } v \in \operatorname{dom}\left(s_{1}\right) . \\
\left(v_{1}, \ldots, v_{\rho(i)}\right) \in R_{i}^{s_{2}} \Leftrightarrow s_{1} \models \varphi_{i}\left[v_{1}, \ldots, v_{\rho(i)}, T_{1}, \ldots, T_{n}\right] \text { for all } i \in I \text { and } \\
\text { all } v_{1}, \ldots, v_{\rho(i)} \in \operatorname{dom}\left(s_{2}\right) .
\end{aligned}
$$

By abusing notation, we define the transduction $\operatorname{def}_{\mathcal{D}} \subseteq \mathcal{C}_{1} \times \mathcal{C}_{2}$ by letting $\left(s_{1}, s_{2}\right) \in \mathbf{d e f}_{\mathcal{D}}$ iff $s_{1} \in \mathcal{C}_{1}$ and there are sets $T_{1}, \ldots, T_{n} \subseteq \operatorname{dom}\left(s_{1}\right)$ with $s_{1} \models \vartheta\left[T_{1}, \ldots, T_{n}\right]$ such that $s_{2}=$ $\operatorname{def}_{\mathcal{D}}\left(s_{1}\right)$. Let us call a definition scheme $\mathcal{D}$ with parameters $X_{1}, \ldots, X_{n}$ unambiguous if for any pair $\left(s_{1}, s_{2}\right) \in \mathbf{d e f}_{\mathcal{D}}$ there is at most one assignment of parameters $\gamma:\left\{X_{1}, \ldots, X_{n}\right\} \rightarrow$ $\mathscr{P}\left(\operatorname{dom}\left(s_{1}\right)\right)$ such that $\operatorname{def}_{\mathcal{D}}\left(s_{1}, \gamma\left(X_{1}\right), \ldots, \gamma\left(X_{n}\right)\right)=s_{2}$.

Definition 5.1. A transduction $\Phi \subseteq \mathcal{C}_{1} \times \mathcal{C}_{2}$ is unambiguously definable if there is a unambiguous definition scheme $\mathcal{D}$ such that $\Phi=\operatorname{def}_{\mathcal{D}}$. It is unambiguously FO-definable if there is an unambiguous definition scheme $\mathcal{D}=\left(\vartheta, \delta,\left(\varphi_{i}\right)_{i \in I}\right)$ defining $\Phi$ with $\vartheta, \delta,\left(\varphi_{i}\right)_{i \in I} \in$ FO.

A transduction which is given by a less restricted definition scheme, where one allows for more than one copy of $s_{1}$ and which is not necessarily unambiguous, is called definable. Courcelle [9] showed that the preimage of a definable set under a definable transduction is again definable. We will show a similar result for series. Let $\Phi: \mathcal{C}_{1} \rightarrow \mathcal{C}_{2}$ be a partial function with domain $\operatorname{dom}(\Phi)$ and let $S: \mathcal{C}_{2} \rightarrow \mathbb{K}$. Define $\Phi^{-1}(S)$ by letting $\left(\Phi^{-1}(S), s_{1}\right)=$ 
$\left(S, \Phi\left(s_{1}\right)\right)$ for all $s_{1} \in \operatorname{dom}(\Phi)$ and $\left(\Phi^{-1}(S), s_{1}\right)=0$ otherwise. If $\Phi$ is injective, we let $\Phi(S)=\left(\Phi^{-1}\right)^{-1}(S)$.

Clearly, $\operatorname{MSO}(\mathbb{K})$ can be defined for $\mathcal{C}_{1}$ and $\mathcal{C}_{2}$ along the same lines as for nested words. In order to disambiguate a formula, we need a linear order on each $s \in \mathcal{C}_{1}$ (resp. $\mathcal{C}_{2}$ ). For the next proposition we therefore assume that there are binary relation symbols $\leq_{1} \in \sigma_{1}$ and $\leq_{2} \in \sigma_{2}$ such that the interpretation of $\leq_{i}$ in $s$ is a linear order for any $s \in \mathcal{C}_{i}(i=1,2)$. Using these linear orders we can define syntactically unambiguous formulae and then $\operatorname{sRMSO}(\mathbb{K})$ and $\operatorname{swRMSO}(\mathbb{K})$ over $\sigma_{1}$ and $\sigma_{2}$.

Proposition 5.2. Let $\Phi: \mathcal{C}_{1} \rightarrow \mathcal{C}_{2}$ be an unambiguously definable partial function. Then the following holds:

(1) If $S: \mathcal{C}_{2} \rightarrow \mathbb{K}$ is $\mathrm{MSO}(\mathbb{K})$-definable, then so is $\Phi^{-1}(S)$.

(2) If $S: \mathcal{C}_{2} \rightarrow \mathbb{K}$ is $\mathrm{sRMSO}(\mathbb{K})$-definable, then so is $\Phi^{-1}(S)$.

(3) If $S: \mathcal{C}_{2} \rightarrow \mathbb{K}$ is swRMSO $(\mathbb{K})$-definable, then so is $\Phi^{-1}(S)$.

(4) If $\Phi$ is unambiguously $\mathrm{FO}$-definable and $S: \mathcal{C}_{2} \rightarrow \mathbb{K}$ is $\operatorname{sREMSO}(\mathbb{K})$-definable, then $\Phi^{-1}(S)$ is sREMSO(K)-definable.

Proof sketch. Full proof and more general results can be found in [30, 31].

Let $\mathcal{D}=\left(\vartheta, \delta,\left(\varphi_{i}\right)_{i \in I}\right)$ be an unambiguous definition scheme defining $\Phi$. Let $\varphi \in \operatorname{MSO}(\mathbb{K})$. By induction on the structure of $\varphi$ we now define the formula $\widehat{\varphi} \in \operatorname{MSO}\left(\mathbb{K}, \sigma_{1}\right)$.

$\widehat{k}=k, \quad \widehat{x=y}=(x=y) \quad \widehat{x \in X}=x \in X \quad R_{i}\left(x_{1}, \ldots, x_{\rho(i)}\right)=\varphi_{i}\left(x_{1} \ldots x_{\rho(i)}, X_{1}, \ldots, X_{n}\right)^{+}$ If $\varphi$ is $x=y, x \in X$ or $R_{i}\left(x_{1}, \ldots, x_{\rho(i)}\right)$ let $\widehat{\neg \psi}=(\widehat{\psi})^{-}$. Moreover, let

$$
\begin{aligned}
& \widehat{\psi_{1} \wedge \psi_{2}}=\widehat{\psi_{1}} \wedge \widehat{\psi_{2}} \\
& \widehat{\psi_{1} \vee \psi_{2}}= \begin{cases}\left(\widehat{\psi_{1}} \vee \widehat{\psi_{2}}\right)^{+} & \text {if } \psi_{1} \vee \psi_{2} \text { is syntactically unambiguous } \\
\widehat{\psi_{1}} \vee \widehat{\psi_{2}} & \text { otherwise }\end{cases} \\
& \widehat{\exists x \cdot \psi}= \begin{cases}{\left[\exists x \cdot\left(\delta\left(x, X_{1}, \ldots, X_{n}\right) \wedge \widehat{\psi}\right)\right]^{+}} & \text {if } \exists x \cdot \psi \text { is syntactically unambiguous } \\
\exists x \cdot\left(\delta\left(x, X_{1}, \ldots, X_{n}\right)^{+} \wedge \widehat{\psi}\right) & \text { otherwise }\end{cases} \\
& \widehat{\exists X . \psi}= \begin{cases}{\left[\exists X . \forall x \cdot\left(x \in X \rightarrow \delta\left(x, X_{1}, \ldots, X_{n}\right)\right) \wedge \widehat{\psi}\right]^{+}} & \text {if } \exists X . \psi \text { is synt. unambiguous } \\
\exists X . \forall x \cdot\left(x \in X \rightarrow \delta\left(x, X_{1}, \ldots, X_{n}\right)\right)^{+} \wedge \widehat{\psi} & \text { otherwise }\end{cases} \\
& \widehat{\forall x \cdot \psi}=\forall x \cdot \delta\left(x, X_{1}, \ldots, X_{n}\right) \stackrel{+}{\rightarrow} \widehat{\psi} \\
& \widehat{\forall X . \psi}=\forall X .\left(\forall x \cdot x \in X \rightarrow \delta\left(x, X_{1}, \ldots, X_{n}\right)\right) \stackrel{+}{\rightarrow} \widehat{\psi} .
\end{aligned}
$$

Now let $\varphi$ be as required such that $\llbracket \varphi \rrbracket=S$. One can show by induction on the structure of $\varphi$ that $\llbracket \exists X_{1}, \ldots X_{n} . \vartheta\left(X_{1}, \ldots, X_{n}\right)^{+} \wedge \widehat{\varphi} \rrbracket=\Phi^{-1}(S)$. By construction we get that if $\varphi$ is syntactically unambiguous, then so is its translation $\widehat{\varphi}$. Again by induction it is therefore

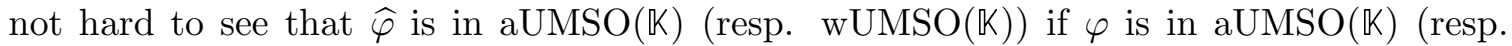
wUMSO$(\mathbb{K}))$. From this we conclude that the translation is as required.

We are now going to show that regular series coincide with $\mathrm{sRMSO}(\mathbb{K})$-definable ones. For this we define two embeddings of nested words into alternating texts and use the characterizations of text series. The connection we establish turns out to be useful again in Section 6. Define $\Phi_{\bullet}, \Phi_{\circ}: \mathrm{NW}(\Delta) \rightarrow \operatorname{TXT}(\Delta)$ as follows. Let $n w=(w, \nu) \in \mathrm{NW}(\Delta)$ where $w=a_{1} \ldots a_{n}$. If $\nu=\emptyset$, then let $\Phi_{\circ}(n w)=a_{1} \circ \ldots \circ a_{n}$ and $\Phi_{\bullet}(n w)=a_{1} \bullet \ldots \bullet a_{n}$. 
If $\nu \neq \emptyset$, let $i$ be the minimal call position and $j$ the corresponding return position. Let $n w^{\prime}=n w[i+1, j-1]$ and $n w^{\prime \prime}=n w[j+1, n]$. Suppose for the moment that $i+1 \leq j-1$ and $j+1 \leq n$. We define

$$
\begin{aligned}
& \Phi_{\circ}(n w)=a_{1} \circ \ldots \circ a_{i-1} \circ\left(a_{i} \bullet \Phi_{\bullet}\left(n w^{\prime}\right) \bullet a_{j}\right) \circ \Phi_{\circ}\left(n w^{\prime \prime}\right), \\
& \Phi_{\bullet}(n w)=a_{1} \bullet \ldots \bullet a_{i-1} \bullet\left(a_{i} \circ \Phi_{\circ}\left(n w^{\prime}\right) \circ a_{j}\right) \bullet \Phi_{\bullet}\left(n w^{\prime \prime}\right) .
\end{aligned}
$$

If $i+1=j$ or $j=n$, then we just ignore the terms $\Phi_{\circ}\left(n w^{\prime}\right), \Phi_{\circ}\left(n w^{\prime \prime}\right), \Phi_{\bullet}\left(n w^{\prime}\right)$ and $\Phi_{\bullet}\left(n w^{\prime \prime}\right)$, respectively, in the definition above. Intuitively, we transform the nesting relation into wellmatched brackets. As an example consider the nested word $n w$ given in Figure 1, Its coding $\Phi_{\circ}(n w)$ is the alternating text in Figure 2.

Let $\Phi_{\circ}(n w)=\left(V^{\circ}, \lambda^{\circ}, \leq_{1}^{\circ}, \leq_{2}^{\circ}\right)$ and $\Phi_{\bullet}(n w)=\left(V^{\bullet}, \lambda^{\bullet}, \leq_{1}^{\bullet}, \leq_{2}^{\bullet}\right)$. The following observations can easily be made by induction either on $n$ or on $|\nu|$ :

(a) Both $V^{\circ}$ and $V^{\bullet}$ have cardinality $n$. We therefore assume from now on that $V^{\circ}=$ $V^{\bullet}=[n]$ such that $\leq_{1}^{0}$ as well as $\leq_{1}^{\bullet}$ is the usual order on $[n]$. It is easy to see that $\lambda^{\circ}(i)=\lambda^{\bullet}(i)=a_{i}$.

(b) Both $\Phi_{\circ}$ and $\Phi_{\bullet}$ are injective.

Recall that a position of $n w$ has odd nesting depth if the number of open call positions is odd (see Example 3.7).

Lemma 5.3. Let $n w=\left(a_{1} \ldots a_{n}, \nu\right) \in \mathrm{NW}(\Delta)$, let $\Phi_{\circ}(n w)=\left([n], \lambda, \leq_{1}^{\circ}, \leq_{2}^{\circ}\right)$ and let $\Phi_{\bullet}(n w)=\left([n], \lambda, \leq_{1}^{\bullet}, \leq_{2}^{\bullet}\right)$. Moreover, let $1 \leq i<j \leq n$. Then we have, $i \geq_{2}^{\circ} j$ iff $i \leq_{2}^{\bullet} j$ iff there is some $(k, \ell) \in \nu$ with $1 \leq k \leq i<j \leq \ell \leq n$ such that there is no $\left(k^{\prime}, \ell^{\prime}\right) \in \nu$ with $k<k^{\prime} \leq i<j \leq \ell^{\prime}<\ell$ and $k$ has odd nesting depth.

Proof. The proof is by induction on $|\nu|$. For $|\nu|=0$ this is trivial. Now let $|\nu| \geq 1$. We only prove that $i \geq_{2}^{0} j$ iff there is some $(k, \ell) \in \nu$ with $1 \leq k \leq i<j \leq \ell \leq n$ such that there is no $\left(k^{\prime}, \ell^{\prime}\right) \in \nu$ with $k<k^{\prime} \leq i<j \leq \ell^{\prime}<\ell$ and $k$ has odd nesting depth. That this holds iff $i \leq_{2}^{\bullet} j$ can be shown analogously. Let $i^{\prime}$ be the minimal call position and $j^{\prime}$ the corresponding return position. Let $n w^{\prime}=n w\left[i^{\prime}+1, j^{\prime}-1\right]$ and $n w^{\prime \prime}=n w\left[j^{\prime}+1, n\right]$ provided they exist. Moreover, let $\Phi_{\bullet}\left(n w^{\prime}\right)=\left(\left[j^{\prime}-i^{\prime}-1\right], \lambda^{\prime}, \leq_{1}^{\prime}, \leq_{2}^{\prime}\right)$ and $\Phi_{\circ}\left(n w^{\prime \prime}\right)=\left(\left[n-j^{\prime}\right], \lambda^{\prime \prime}, \leq_{1}^{\prime \prime}, \leq_{2}^{\prime \prime}\right)$. We consider three cases:

(1) Assume $i<i^{\prime}$ or $i \leq j^{\prime}<j$. Then $i \leq_{2}^{\circ} j$ and there is no $(k, \ell) \in \nu$ with $1 \leq k \leq i<$ $j \leq l \leq n$.

(2) Assume $i^{\prime} \leq i<j \leq j^{\prime}$. If $i=i^{\prime}$ or $j=j^{\prime}$, then $i \geq_{2}^{0} j$ and choosing $(k, \ell)=(i, j)$ gives $(k, \ell)$ as required since $i$ has nesting depth 1 . If $i^{\prime}<i<j<j^{\prime}$, then we get:

$$
\begin{aligned}
i \geq_{2}^{\circ} j & \Longleftrightarrow \quad i-i^{\prime} \geq_{2}^{\prime} j-i^{\prime} \\
& \Longleftrightarrow \operatorname{not} i-i^{\prime} \leq_{2}^{\prime} j-i^{\prime}
\end{aligned}
$$

either there is some $(k, \ell) \in \nu\left[i^{\prime}+1, j^{\prime}-1\right]$ with $1 \leq$ $k \leq i-i^{\prime}<j-i^{\prime} \leq \ell \leq j^{\prime}-i^{\prime}-1$ such that there is no

$\Longleftrightarrow \quad\left(k^{\prime}, \ell^{\prime}\right) \in \nu\left[i^{\prime}+1, j^{\prime}-1\right]$ with $k<k^{\prime} \leq i-i^{\prime}<j-i^{\prime} \leq \ell^{\prime}<\ell$ and $k$ has even nesting depth in $n w^{\prime}$, or there is no $(k, \ell) \in$ $\nu\left[i^{\prime}+1, j^{\prime}-1\right]$ with $1 \leq k \leq i-i^{\prime}<j-i^{\prime} \leq \ell \leq j^{\prime}-i^{\prime}-1$

there is some $(k, \ell) \in \nu$ with $1 \leq k \leq i<j \leq \ell \leq n$ such

$\Longleftrightarrow \quad$ that there is no $\left(k^{\prime}, \ell^{\prime}\right) \in \nu$ with $k<k^{\prime} \leq i<j \leq \ell^{\prime}<\ell$ and $k$ has odd nesting depth. 
(3) Assume $j^{\prime}<i$. Then we get

$$
\begin{aligned}
i \geq_{2}^{\circ} j \Longleftrightarrow \quad & i-j^{\prime} \geq_{2}^{\prime \prime} j-j^{\prime} \\
& \text { there is some }(k, \ell) \in \nu\left[j^{\prime}+1, n\right] \text { with } 1 \leq k \leq i-j^{\prime}< \\
& j-j^{\prime} \leq \ell \leq n-j^{\prime} \text { such that there is no }\left(k^{\prime}, \ell^{\prime}\right) \in \nu\left[j^{\prime}+1, n\right] \\
& \text { with } k<k^{\prime} \leq i-j^{\prime}<j-j^{\prime} \leq \ell^{\prime}<\ell \text { and } k \text { has odd } \\
& \text { nesting depth } \\
& \text { there is some }(k, \ell) \in \nu \text { with } 1 \leq k \leq i<j \leq \ell \leq n \text { such } \\
& \text { that there is no }\left(k^{\prime}, \ell^{\prime}\right) \in \nu \text { with } k<k^{\prime} \leq i<j \leq \ell^{\prime}<\ell \\
& \text { and } k \text { has odd nesting depth. }
\end{aligned}
$$

Corollary 5.4. The functions $\Phi_{\circ}$ and $\Phi_{\bullet}$ are unambiguously FO-definable.

Proof. We only show that $\Phi_{\circ}$ is FO-definable. For $\Phi_{\bullet}$ the claim can be shown analogously. We give a 1-copying definition scheme $\left(\vartheta, \delta,\left(\varphi_{\mathrm{Lab}_{a}}\right)_{a \in \Delta}, \varphi_{\leq_{1}}, \varphi_{\leq_{2}}\right)$ with four parameters $X_{1}, X_{2}, Y_{1}, Y_{2}$.

Let the macros call $(x)$ and return $(x)$ be as in Example 3.7. Moreover, let

$$
\operatorname{Frst}_{\nu}(x)=\operatorname{call}(x) \wedge \forall y \cdot \operatorname{call}(y) \rightarrow x \leq y
$$

The next macro defines $y$, the next call or return position following position $x$.

$$
\operatorname{next}_{\nu}(x, y)=x<y \wedge(\operatorname{call}(y) \vee \operatorname{return}(y)) \wedge \forall z .(x<z<y) \rightarrow(\neg \operatorname{call}(z) \wedge \neg \operatorname{return}(z))
$$

We now define the formula $\vartheta\left(X_{1}, X_{2}, Y_{1}, Y_{2}\right)$ which for all $n w=\left(a_{1} \ldots a_{n}, \nu\right) \in \mathrm{NW}(\Delta)$ and $C_{1}, C_{2}, R_{1}, R_{2} \subseteq[n]$ has the property that $n w \models \vartheta\left[C_{1}, C_{2}, R_{1}, R_{2}\right]$ iff $C_{1}$ is the set of all call positions of odd nesting depth, $C_{2}$ is the set of all call positions of even nesting depth, $R_{1}$ is the set of all return positions of even nesting depth and $R_{2}$ is the set of all return positions of odd nesting depth.

$$
\begin{aligned}
\vartheta\left(X_{1}, X_{2}, Y_{1}, Y_{2}\right)= & \left(X_{1} \cap X_{2}=\emptyset\right) \wedge \forall z \cdot\left(z \in X_{1} \vee z \in X_{2}\right) \rightarrow \operatorname{call}(z) \\
& \wedge\left(Y_{1} \cap Y_{2}=\emptyset\right) \wedge \forall z \cdot\left(z \in Y_{1} \vee z \in Y_{2}\right) \rightarrow \operatorname{return}(z) \\
& \wedge \forall z \cdot \operatorname{Frst}_{\nu}(z) \rightarrow z \in X_{1} \\
& \wedge \forall z_{1}, z_{2} \cdot\left(\left(z_{1} \in X_{1} \wedge \operatorname{next}_{\nu}\left(z_{1}, z_{2}\right) \wedge \operatorname{return}\left(z_{2}\right)\right) \rightarrow z_{2} \in Y_{1}\right) \\
& \wedge \forall z_{1}, z_{2} \cdot\left(\left(z_{1} \in X_{1} \wedge \operatorname{next}_{\nu}\left(z_{1}, z_{2}\right) \wedge \operatorname{call}\left(z_{2}\right)\right) \rightarrow z_{2} \in X_{2}\right) \\
& \wedge \forall z_{1}, z_{2} \cdot\left(\left(z_{1} \in X_{2} \wedge \operatorname{next}_{\nu}\left(z_{1}, z_{2}\right) \wedge \operatorname{return}\left(z_{2}\right)\right) \rightarrow z_{2} \in Y_{2}\right) \\
& \wedge \forall z_{1}, z_{2} \cdot\left(\left(z_{1} \in X_{2} \wedge \operatorname{next}_{\nu}\left(z_{1}, z_{2}\right) \wedge \operatorname{call}\left(z_{2}\right)\right) \rightarrow z_{2} \in X_{1}\right) \\
& \wedge \forall z_{1}, z_{2} \cdot\left(\left(z_{1} \in Y_{1} \wedge \operatorname{next}_{\nu}\left(z_{1}, z_{2}\right) \wedge \operatorname{return}\left(z_{2}\right)\right) \rightarrow z_{2} \in Y_{2}\right) \\
& \wedge \forall z_{1}, z_{2} \cdot\left(\left(z_{1} \in Y_{1} \wedge \operatorname{next}_{\nu}\left(z_{1}, z_{2}\right) \wedge \operatorname{call}\left(z_{2}\right)\right) \rightarrow z_{2} \in X_{1}\right) \\
& \wedge \forall z_{1}, z_{2} \cdot\left(\left(z_{1} \in Y_{2} \wedge \operatorname{next}_{\nu}\left(z_{1}, z_{2}\right) \wedge \operatorname{return}\left(z_{2}\right)\right) \rightarrow z_{2} \in Y_{1}\right) \\
& \wedge \forall z_{1}, z_{2} \cdot\left(\left(z_{1} \in Y_{2} \wedge \operatorname{next}_{\nu}\left(z_{1}, z_{2}\right) \wedge \operatorname{call}\left(z_{2}\right)\right) \rightarrow z_{2} \in X_{2}\right)
\end{aligned}
$$

where $X \cap Y=\emptyset$ abbreviates $\neg(\exists z . z \in X \wedge z \in Y)$. We let $\delta\left(x, X_{1}, X_{2}, Y_{1}, Y_{2}\right)$ be some tautology. Now we define the interpreting formulae. We set $\left.\varphi_{\mathrm{Lab}_{a}}\left(x, X_{1}, X_{2}, Y_{1}, Y_{2}\right)\right)=$ $\operatorname{Lab}_{a}(x)$ and let $\left.\varphi_{\leq_{1}}\left(x, y, X_{1}, X_{2}, Y_{1}, Y_{2}\right)\right)=x \leq y$. Furthermore, we define $\varphi_{\circ}\left(x, y, X_{1}\right)$ to 
be the following formula which expresses the condition of Lemma 5.3 ,

$$
\begin{aligned}
\varphi_{\circ}\left(x, y, X_{1}\right)=[x<y \wedge & \left(\exists z_{1}, z_{2} \cdot\left(z_{1} \leq x \leq y \leq z_{2}\right) \wedge \nu\left(z_{1}, z_{2}\right) \wedge z_{1} \in X_{1} \wedge\right. \\
& \left.\left.\wedge \forall z_{1}^{\prime}, z_{2}^{\prime} \cdot\left(z_{1}<z_{1}^{\prime} \leq x \leq y \leq z_{2}^{\prime}<z_{2}\right) \rightarrow \neg \nu\left(z_{1}^{\prime}, z_{2}^{\prime}\right)\right)\right]
\end{aligned}
$$

and let

$$
\varphi_{\leq_{2}}\left(x, y, X_{1}\right)=x=y \vee\left(y<x \wedge \varphi_{\circ}\left(y, x, X_{1}\right)\right) \vee\left(x<y \wedge \neg \varphi_{\circ}\left(x, y, X_{1}\right)\right) .
$$

This completes the definition scheme for $\Phi_{\circ}$ which is unambiguous.

Let $\tau=\left([n], \lambda, \leq_{1}, \leq_{2}\right)$ be a text. An interval $[i, j]=\left\{k \in[n] \mid i \leq_{1} k \leq_{1} j\right\}$ of the first order is a clan if it is an interval also of the second order. A prime clan is a clan that does not overlap with any other, i.e. there is no clan $[k, \ell]$ such that $k<_{1} i<_{1} \ell<_{1} j$ or $i<_{1} k<_{1} j<_{1} \ell$.

Lemma 5.5. Let $n w=\left(a_{1} \ldots a_{n}, \nu\right) \in \mathrm{NW}(\Delta)$, let $\Phi_{\circ}(n w)=\left([n], \lambda, \leq_{1}^{\circ}, \leq_{2}^{\circ}\right)$ and let $\Phi_{\bullet}(n w)=\left([n], \lambda, \leq_{1}^{\bullet}, \leq_{2}^{\bullet}\right)$. Moreover, let $1 \leq i<j \leq n$.

Then $(i, j) \in \nu$ iff $[i, j]$ is a prime clan of $\Phi_{\circ}(n w)$ and we have either $i \neq 1, j \neq n$ or $1 \geq_{2}^{\circ} n$ iff $[i, j]$ is a prime clan of $\Phi_{\bullet}(n w)$ and we have either $i \neq 1, j \neq n$ or $1 \leq_{2}^{\bullet} n$.

Proof. The proof is again by induction on $|\nu|$. If $\nu=\emptyset$, then $[1, n]$ is the only prime clan of both $\Phi_{\circ}(n w)$ and $\Phi_{\bullet}(n w)$ (since any other clan can be overlapped) and we have $1 \leq_{2}^{\circ} n$ and $1 \geq \geq_{2}^{\bullet} n$. Now let $|\nu| \geq 1$ and let $\left(i_{1}, j_{1}\right),\left(i_{2}, j_{2}\right), \ldots,\left(i_{t}, j_{t}\right)$ with $i_{1}<i_{2}<\ldots<i_{t}$ be the sequence of surface arches (see definition after Def. 2.11). By definition we have

$$
\begin{aligned}
& \Phi_{\circ}(n w)=\Phi_{\circ}\left(n w\left[1, i_{1}-1\right]\right) \circ \Phi_{\circ}\left(n w\left[i_{1}, j_{1}\right]\right) \circ \cdots \circ \\
& \circ \Phi_{\circ}\left(n w\left[j_{t-1}+1, i_{t}-1\right]\right) \circ \Phi_{\circ}\left(n w\left[i_{t}, j_{t}\right]\right) \circ \Phi_{\circ}\left(n w\left[j_{t}+1, n\right]\right) \text {, }
\end{aligned}
$$

where we ignore a factor if the corresponding interval is empty. We show that $(i, j) \in \nu$ iff $[i, j]$ is a prime clan of $\Phi_{\circ}(n w)$ and we have either $i \neq 1, j \neq n$ or $1 \geq_{2}^{0} n$. That this holds iff $[i, j]$ is a prime clan of $\Phi_{\bullet}(n w)$ and we have either $i \neq 1, j \neq n$ or $1 \leq_{2}^{\bullet} n$ can again be shown analogously.

(Only if). Let $(i, j) \in \nu$. Then there is some $r$ such that $i_{r} \leq i<j \leq j_{r}$.

If $i=i_{r}$ or $j=j_{r}$, then $i=i_{r}$ and $j=j_{r}$. Clearly, $\left[i_{r}, j_{r}\right]$ is a clan. Suppose for contradiction that there is a clan $[\ell, k]$ overlapping $\left[i_{r}, j_{r}\right]$. Assume $\ell<i_{r}<k<j_{r}$ (the case $i_{r}<\ell<j_{r}<k$ is similar). By definition of $\Phi_{\circ}$ we get $\ell \leq_{2}^{\circ} j_{r} \leq_{2}^{\circ} i_{r}$. Contradiction. Thus $\left[i_{r}, j_{r}\right]$ is a prime clan. In particular if $i_{r}=1$ and $j_{r}=n$, we get $1 \geq_{2}^{\circ} n$.

Otherwise, in case of $i_{r}<i<j<j_{r}$, the interval $\left[i-i_{r}, j-i_{r}\right]$ is a prime clan of $\Phi .\left(n w\left[i_{r}+1, j_{r}-1\right]\right)$ by induction hypothesis. Thus, $[i, j]$ must be a clan, since $\left[i_{r}, j_{r}\right]$ is a clan, too. Suppose for contradiction that there is a clan $[\ell, k]$ overlapping $[i, j]$. As $\left[i-i_{r}, j-i_{r}\right]$ is a prime clan of $\Phi_{\bullet}\left(n w\left[i_{r}+1, j_{r}-1\right]\right)$ we get either $\ell \leq i_{r}$ or $k \geq j_{r}$. Assume $\ell \leq i_{r}$ (the other case is similar). Now, if $\ell<i_{r}$, we can argue as above and separate $\ell$ and $i_{r}$. Contradiction. If $i_{r}=\ell$ and $i_{r}+1<i$, then $\left[1, k-i_{r}\right]$ is a clan in $\Phi_{\bullet}\left(n w\left[i_{r}+1, j_{r}-1\right]\right)$ which overlaps $\left[i-i_{r}, j-i_{r}\right]$. Contradiction. And if $\ell=i_{r}$ and $i_{r}+1=i$, we get by definition $i \leq_{2}^{\circ} j \leq_{2}^{\circ} i_{r}$. Again contradiction. Thus $[i, j]$ must be a prime clan.

(If ). Let $[i, j]$ be a prime clan such that not $i=1, j=n$ and $1 \leq_{2}^{\circ} n$. If $i=1$ and $j=n$, then $1 \geq_{2}^{\circ} n$ and $(i, j) \in \nu$ by definition of $\Phi_{\circ}$. Now suppose $1<i$ or $j<n$. The following intervals (provided they exist) can easily seen to be clans: [1, $\left.i_{1}-1\right],\left[i_{1}, j_{1}\right],\left[j_{1}+1, n\right],\left[1, j_{1}\right]$ and $[\ell, n]$ for any $\ell \leq i_{1}$. From this we conclude that either $i_{1} \leq i<j \leq j_{1}$ or $j_{1}<i$ since otherwise one of the clans above would overlap $[i, j]$. If $i=i_{1}$ or $j=j_{1}$ then $i=i_{1}$ and 
$j=j_{1}$, since $\left[i_{1}, j_{1}-1\right]$ and $\left[i_{1}+1, j_{1}\right]$ are clans, and hence $(i, j) \in \nu$. In the case where $i_{1}<i<j<j_{1}$, we get that $\left[i-i_{1}, j-i_{1}\right]$ must be a prime clan of $\Phi_{\bullet}\left(n w\left[i_{1}+1, j_{1}-1\right]\right)$ and if $j_{1}<i$, we get that $\left[i-j_{1}, j-j_{1}\right]$ must be a prime clan of $\Phi_{\circ}\left(n w\left[j_{1}+1, n\right]\right)$. Hence, in both cases $(i, j) \in \nu$ by induction hypothesis.

It is not hard to see that the domains of the partial functions $\Phi_{\circ}^{-1}$ and $\Phi_{\bullet}^{-1}$ are FOdefinable. Hence, by the last lemma there is a definition scheme without parameters consisting of FO-formulae which defines $\Phi_{\circ}^{-1}$ (or alternatively $\Phi_{\bullet}^{-1}$ ).

Corollary 5.6. The partial functions $\Phi_{\circ}^{-1}$ and $\Phi_{\bullet}^{-1}$ are unambiguously $\mathrm{FO}$-definable.

So far we have seen that we can translate a formula over nested words into a formula over texts (and vice versa) such that the formulae correspond to each other with respect to $\Phi_{\circ}$ resp. $\Phi_{\bullet}$. We will now show that also WPA can simulate WNWA (and vice versa) with respect to $\Phi_{\circ}$ resp. $\Phi_{\bullet}$.

Proposition 5.7. Let $S: \operatorname{TXT}(\Delta) \rightarrow \mathbb{K}$ be regular. Then $\Phi_{\circ}^{-1}(S), \Phi_{\bullet}^{-1}(S): \mathrm{NW}(\Delta) \rightarrow \mathbb{K}$ are regular.

Proof. We show that $\Phi_{\circ}^{-1}(S)$ is regular. Analogously one can show that $\Phi_{\bullet}^{-1}(S)$ is regular. Let $\mathcal{P}=\left(\mathcal{H}, \mathcal{V}, \Omega, \mu, \mu_{\mathrm{op}}, \mu_{\mathrm{cl}}, \lambda, \gamma\right)$ be a WPA such that $\|\mathcal{P}\|=S$. We construct a WNWA $\mathcal{A}=(Q, \iota, \delta, \kappa)$ with state space $Q=(\mathcal{H} \uplus \mathcal{V}) \times(\Omega \uplus\{i\})$ such that for all $h_{0}, h_{n} \in \mathcal{H}$, $v_{0}, v_{n} \in \mathcal{V}$ and $\omega \in \Omega \uplus\{i\}$ we have

$$
\sum_{r:\left(h_{0}, \omega\right) \stackrel{n w}{\longrightarrow}\left(h_{n}, \omega\right)} \operatorname{wgt}_{\mathcal{A}}(r)=\sum_{r: h_{0} \stackrel{\Phi_{o}(n w)}{\longrightarrow} h_{n}} \operatorname{wgt}_{\mathcal{P}}(r) \quad \text { and } \sum_{r:\left(v_{0}, \omega\right) \stackrel{n w}{\longrightarrow}\left(v_{n}, \omega\right)} \operatorname{wgt}_{\mathcal{A}}(r)=\sum_{r: v_{0} \stackrel{\Phi_{\bullet}(n w)}{\longrightarrow} v_{n}} \operatorname{wgt}_{\mathcal{P}}(r) .
$$

Intuitively, in the first component one simulates the states of the WPA and in the second component one stores the most recent open bracket. This has to be updated when reading a return position using the look-back ability of the WNWA. We give now the formal definition of the transition functions. We give it only on certain subsets of their domains. In all other cases we set the values to 0 . Let $a \in \Delta, h_{1}, h_{2} \in \mathcal{H}, v_{1}, v_{2} \in \mathcal{V}, \omega_{1} \in \Omega \uplus\{i\}$ and $\omega_{2} \in \Omega$. Define

$$
\begin{aligned}
\delta_{\text {int }}\left(\left(h_{1}, \omega_{1}\right), a,\left(h_{2}, \omega_{1}\right)\right) & =\mu\left(h_{1}, a, h_{2}\right) \\
\delta_{\text {int }}\left(\left(v_{1}, \omega_{1}\right), a,\left(v_{2}, \omega_{1}\right)\right) & =\mu\left(v_{1}, a, v_{2}\right) \\
\delta_{\text {call }}\left(\left(h_{1}, \omega_{1}\right), a,\left(v_{1}, \omega_{2}\right)\right) & =\sum_{v \in \mathcal{V}} \mu_{\mathrm{op}}\left(h_{1},\left(\omega_{2}, v\right) \cdot \mu\left(v, a, v_{1}\right)\right. \\
\delta_{\text {call }}\left(\left(v_{1}, \omega_{1}\right), a,\left(h_{1}, \omega_{2}\right)\right) & =\sum_{h \in \mathcal{H}} \mu_{\mathrm{op}}\left(v_{1},\left(\omega_{2}, h\right) \cdot \mu\left(h, a, h_{1}\right)\right. \\
\delta_{\text {ret }}\left(\left(h_{1}, \omega_{2}\right),\left(v_{1}, \omega_{1}\right), a,\left(v_{2}, \omega_{1}\right)\right) & \left.=\sum_{h \in \mathcal{H}} \mu\left(h_{1}, a, h\right) \cdot \mu_{\mathrm{cl}}(h,)_{\omega_{2}}, v_{2}\right) \\
\delta_{\text {ret }}\left(\left(v_{1}, \omega_{2}\right),\left(h_{1}, \omega_{1}\right), a,\left(h_{2}, \omega_{1}\right)\right) & \left.=\sum_{v \in \mathcal{V}} \mu\left(v_{1}, a, v\right) \cdot \mu_{\mathrm{cl}}(v,)_{\omega_{2}}, h_{2}\right) .
\end{aligned}
$$

Observe that for any $n w \in \mathrm{NW}(\Delta)$ and any run $r: q_{0} \stackrel{n w}{\longrightarrow} q_{n}$ of $\mathcal{A}$ such that $\operatorname{wgt}_{\mathcal{A}}(r) \neq 0$ the second components of $q_{0}$ and $q_{n}$ coincide and the first components are either both in $\mathcal{H}$ or both in $\mathcal{V}$. 
Let $n w=\left(a_{1} \ldots a_{n}, \nu\right)$. We show Equation 5.1 by induction on $|\nu|$. First let $\nu=\emptyset$. Then for all $h_{0}, h_{n} \in \mathcal{H}$ and $\omega \in \Omega \uplus\{i\}$ we have

$$
\begin{aligned}
\sum_{r:\left(h_{0}, \omega\right) \stackrel{n w}{\longrightarrow}\left(h_{n}, \omega\right)} \operatorname{wgt}_{\mathcal{A}}(r) & =\sum_{h_{1}, \ldots, h_{n-1} \in \mathcal{H}} \prod_{j=1}^{n} \delta_{\text {int }}\left(\left(h_{j-1}, \omega\right), a_{j},\left(h_{j}, \omega\right)\right)= \\
& =\sum_{h_{1}, \ldots, h_{n-1} \in \mathcal{H}} \prod_{j=1}^{n} \mu\left(h_{j-1}, a_{j}, h_{j}\right)=\sum_{r: h_{0} \stackrel{\Phi_{\circ}(n w)}{\longrightarrow} h_{n}} \operatorname{wgt}_{\mathcal{P}}(r) .
\end{aligned}
$$

Similarly we get the claim for $\Phi_{\bullet}$. Now, let $\nu \neq \emptyset$, let $k$ be the minimal call position and let $\ell$ be the corresponding return position. Let $n w_{1}=n w[1, k-1], n w_{2}=n w[k+1, \ell-1]$ and $n w_{3}=n w[\ell+1, n]$ (we assume that all nested words exist, the cases where they do not exist are similar). Then for all $h_{0}, h_{n} \in \mathcal{H}$ and $\omega \in \Omega \uplus\{i\}$ we have

$$
\begin{aligned}
& \sum_{r:\left(h_{0}, \omega\right) \stackrel{n w}{\longrightarrow}\left(h_{n}, \omega\right)} \operatorname{wgt}_{\mathcal{A}}(r)= \\
& =\sum_{\substack{h_{k-1}, h_{\ell} \in \mathcal{H} \\
v_{k}, v_{\ell-1} \in \mathcal{V} \\
\omega_{1} \in \Omega}} \sum_{r_{1}:\left(h_{0}, \omega\right) \stackrel{n w_{1}}{\longrightarrow}\left(h_{k-1}, \omega\right)} \operatorname{wgt}_{\mathcal{A}}\left(r_{1}\right) \cdot \delta_{\text {call }}\left(\left(h_{k-1}, \omega\right), a_{k},\left(v_{k}, \omega_{1}\right)\right) . \\
& \cdot \sum_{r_{2}:\left(v_{k}, \omega_{1}\right) \stackrel{n w_{2}}{\longrightarrow}\left(v_{\ell-1}, \omega_{1}\right)} \operatorname{wgt}_{\mathcal{A}}\left(r_{2}\right) \cdot \delta_{\mathrm{ret}}\left(\left(v_{\ell-1}, \omega_{1}\right),\left(h_{k-1}, \omega\right), a_{\ell},\left(h_{\ell}, \omega\right)\right) \cdot \sum_{r_{3}:\left(h_{\ell}, \omega\right) \stackrel{n w_{3}}{\longrightarrow}\left(h_{n}, \omega\right)} \operatorname{wgt}_{\mathcal{A}}\left(r_{3}\right) \\
& =\sum_{\substack{h_{k-1}, h_{\ell} \in \mathcal{H} \\
v_{k}, v_{\ell-1} \in \mathcal{V}}} \sum_{\substack{r_{1}: h_{0} \\
a_{1} \circ \ldots \circ a_{k-1}}} \operatorname{wgt}_{\mathcal{P}}\left(r_{1}\right) \cdot \sum_{v \in \mathcal{V}} \mu_{\mathrm{op}}\left(h_{k-1},\left(\omega_{1}, v\right) \cdot \mu\left(v, a_{k}, v_{k}\right) \cdot\right. \\
& \omega_{1} \in \Omega \\
& \left.\cdot \sum_{r_{2}: v_{k} \stackrel{\Phi_{\bullet}\left(n w_{2}\right)}{\longrightarrow} v_{\ell-1}} \operatorname{wgt}_{\mathcal{P}}\left(r_{2}\right) \cdot \sum_{v^{\prime} \in \mathcal{V}} \mu\left(v_{\ell-1}, a_{l}, v^{\prime}\right) \cdot \mu_{\mathrm{cl}}\left(v^{\prime},\right)_{\omega_{1}}, h_{\ell}\right) \cdot \sum_{r_{3}: h_{\ell} \stackrel{\Phi_{\circ}\left(n w_{3}\right)}{\longrightarrow} h_{n}} \operatorname{wgt}_{\mathcal{P}}\left(r_{3}\right) \\
& =\sum_{r: h_{0} \stackrel{\Phi_{0}(n w)}{\longrightarrow} h_{n}} \operatorname{wgt}_{\mathcal{P}}(r) \text {. }
\end{aligned}
$$

Again, the claim is shown similarly for $\Phi_{\bullet}$. This concludes the proof of Equation (5.1).

Now consider the WNWA with states $Q^{\prime}=\{\perp, ?, s, \circ, \bullet\}$ and transition functions $\delta_{\text {call }}^{\prime}, \delta_{\text {int }}^{\prime}, \delta_{\text {ret }}^{\prime}$ given for all $a \in \Delta$ and $p \in Q^{\prime} \backslash\{\perp\}$ by

$$
\begin{aligned}
& \delta_{\text {call }}^{\prime}(\perp, a, ?)=\delta_{\text {int }}^{\prime}(\perp, a, s)=\delta_{\text {call }}^{\prime}(s, a, \circ)=\delta_{\text {int }}^{\prime}(s, a, \circ)=\delta_{\text {call }}^{\prime}(?, a, ?)=\delta_{\text {int }}^{\prime}(?, a, ?)= \\
& =\delta_{\text {ret }}^{\prime}(?, p, a, ?)=\delta_{\text {ret }}^{\prime}(?, \perp, a, \bullet)=\delta_{\text {call }}^{\prime}(\bullet, a, \circ)=\delta_{\text {int }}^{\prime}(\bullet, a, \circ)=\delta_{\text {call }}^{\prime}(\circ, a, \circ)= \\
& =\delta_{\text {int }}^{\prime}(\circ, a, \circ)=\delta_{\text {ret }}^{\prime}(\circ, p, a, \circ)=1 .
\end{aligned}
$$

Set any other values of $\delta_{\text {call }}^{\prime}, \delta_{\text {int }}^{\prime}, \delta_{\text {ret }}^{\prime}$ to 0 and let the initial distribution $\iota^{\prime}$ be given by $\iota^{\prime}\left(q^{\prime}\right)=1$ if $q^{\prime}=\perp$ and 0 otherwise. Observe that in the case where the final distribution $\kappa^{\prime}$ is given by $\kappa^{\prime}\left(q^{\prime}\right)=1$ if $q^{\prime}=0$ and 0 otherwise, the behavior of the automaton is the characteristic series of the set of nested words $n w$ such that $\Phi_{\circ}(n w)$ is a o-product. We collect such nested words in $\mathrm{NW}^{\circ}$. In the case where the final distribution $\kappa^{\prime}$ is given by $\kappa^{\prime}\left(q^{\prime}\right)=1$ if $q^{\prime}=\bullet$ and 0 otherwise, the behavior of the automaton is the characteristic series of the set of nested words $n w$ such that $\Phi_{\circ}(n w)$ is a $\bullet$-product. We collect such nested 
words in $\mathrm{NW}^{\bullet}$. Finally, in the case where the final distribution $\kappa^{\prime}$ is given by $\kappa^{\prime}\left(q^{\prime}\right)=1$ if $q^{\prime}=s$ and 0 otherwise, the behavior of the automaton is the characteristic series of the set of all singleton nested words, i.e. $\Delta$.

Now consider the product of this automaton with $\mathcal{A}$ which has states $Q \times Q^{\prime}$ and whose transition functions $\delta_{\text {call }}^{\times}, \delta_{\text {int }}^{\times}, \delta_{\text {ret }}^{\times}$is given by letting $\delta_{\text {call }}^{\times}\left(\left(q, q^{\prime}\right), a,\left(p, p^{\prime}\right)=\delta_{\text {call }}(q, a, p)\right.$. $\delta\left(q^{\prime}, a, p^{\prime}\right)$ for all $q, p \in Q$ and $q^{\prime}, p^{\prime} \in Q^{\prime}$. If we define the initial and final distribution $\iota^{\times}$ and $\kappa^{\times}$by letting for all $h \in \mathcal{H}$ and $\omega \in \Omega$

$$
\begin{aligned}
\iota^{\times}((h, i), \perp) & =\lambda(h) & \iota((h, \omega), \perp) & =\sum_{v \in \mathcal{V}} \lambda(v) \cdot \mu_{\mathrm{op}}\left(v,\left({ }_{\omega}, h\right)\right. \\
\kappa((h, i), \circ) & =\gamma(h) & \kappa((h, i), \circ) & \left.=\sum_{v \in \mathcal{V}} \mu_{\mathrm{cl}}(h,)_{\omega}, v\right) \cdot \gamma(v),
\end{aligned}
$$

and in any other case by setting the value to 0 , then the behavior of the resulting automaton is $\mathbb{1}_{\mathrm{NW}^{\circ}} \odot \Phi_{\circ}^{-1}(S)$. Changing the definitions of $\iota^{\times}, \kappa^{\times}$appropriately gives automata with behavior $\mathbb{1}_{\mathrm{NW}} \bullet \odot \Phi_{\circ}^{-1}(S)$ and $\mathbb{1}_{\Delta} \odot \Phi_{\circ}^{-1}(S)$. The automaton obtained from disjoint copies of these three automata has hence the behavior $\Phi_{\circ}^{-1}(S)$.

Proposition 5.8. Let $S: \mathrm{NW}(\Delta) \rightarrow \mathbb{K}$ be a regular series. Then $\Phi_{\circ}(S), \Phi_{\bullet}(S): \operatorname{TXT}(\Delta) \rightarrow$ KK are regular.

Proof. Let $\mathcal{A}=(Q, \iota, \delta, \kappa)$ be a WNWA. We define a WPA $\mathcal{P}=\left(\mathcal{H}, \mathcal{V}, \Omega, \mu, \mu_{\mathrm{op}}, \mu_{\mathrm{cl}}, \lambda, \gamma\right)$ with

$$
\mathcal{H}=\left\{q^{\mathcal{H}} \mid q \in Q\right\} \times(\{c, i\} \uplus \Delta) \quad \text { and } \quad \mathcal{V}=\left\{q^{\mathcal{V}} \mid q \in Q\right\} \times(\{c, i\} \uplus \Delta)
$$

as well as $\Omega=Q$ such that $\left(\|\mathcal{P}\|, \Phi_{\circ}(n w)\right)=(\|\mathcal{A}\|, n w)$ for all $n w \in \mathrm{NW}(\Delta)$. To prove the result for $\Phi$. only $\lambda$ and $\gamma$ have to be changed.

Intuitively, in the first component one simulates the states of the WNWA, in the second component one either selects whether the next transition is a call or an internal transition, or one stores the letter to simulate a return position with the next bracket. Look-back behavior is simulated by storing a state in the opening bracket and closing it at the appropriate return position.

We formally define $\mu, \mu_{\mathrm{op}}, \mu_{\mathrm{cl}}$ as follows. We give the definition only on certain subsets of their domains. In all other cases we set their values to 0 .

$$
\begin{aligned}
\mu\left(\left(q_{1}^{\mathcal{H}}, i\right), a,\left(q_{2}^{\mathcal{H}}, i\right)\right) & =\delta_{\text {int }}\left(q_{1}, a, q_{2}\right) & \mu\left(\left(q_{1}^{\mathcal{V}}, i\right), a,\left(q_{2}^{\mathcal{V}}, i\right)\right) & =\delta_{\text {int }}\left(q_{1}, a, q_{2}\right) \\
\mu\left(\left(q_{1}^{\mathcal{H}}, c\right), a,\left(q_{2}^{\mathcal{H}}, i\right)\right) & =\delta_{\text {call }}\left(q_{1}, a, q_{2}\right) & \mu\left(\left(q_{1}^{\mathcal{V}}, c\right), a,\left(q_{2}^{\mathcal{V}}, i\right)\right) & =\delta_{\text {call }}\left(q_{1}, a, q_{2}\right) \\
\mu\left(\left(q_{1}^{\mathcal{H}}, i\right), a,\left(q_{1}^{\mathcal{H}}, a\right)\right) & =1 & \mu\left(\left(q_{1}^{\mathcal{V}}, i\right), a,\left(q_{1}^{\mathcal{V}}, a\right)\right) & =1 \\
\mu_{\mathrm{op}}\left(\left(q_{1}^{\mathcal{H}}, i\right),\left(_{q_{1}},\left(q_{1}^{\mathcal{V}}, c\right)\right)\right. & =1 & \mu_{\mathrm{op}}\left(\left(q_{1}^{\mathcal{V}}, i\right),\left(q_{1},\left(q_{1}^{\mathcal{H}}, c\right)\right)\right. & =1 \\
\left.\mu_{\mathrm{cl}}\left(\left(q_{1}^{\mathcal{H}}, a\right),\right)_{q_{2}},\left(q_{3}^{\mathcal{V}}, i\right)\right) & =\delta_{\text {ret }}\left(\left(q_{1}, q_{2}, a, q_{3}\right)\right. & \left.\mu_{\mathrm{cl}}\left(\left(q_{1}^{\mathcal{V}}, a\right),\right)_{q_{2}},\left(q_{3}^{\mathcal{H}}, i\right)\right) & =\delta_{\text {ret }}\left(\left(q_{1}, q_{2}, a, q_{3}\right)\right. \\
\lambda\left(q_{1}^{\mathcal{H}}, i\right) & =\iota\left(q_{1}\right) & \gamma\left(q_{1}^{\mathcal{H}}, i\right) & =\gamma\left(q_{1}\right)
\end{aligned}
$$

We use induction on $n w=\left(a_{1} \ldots a_{n}, \nu\right) \in \mathrm{NW}(\Delta)$ to show that the defined WPA behaves as required. More precisely we show that for all $q_{1}, q_{2} \in Q$

$$
\sum_{r:\left(q_{1}^{\mathcal{H}}, i\right) \stackrel{\Phi_{0}(n w)}{\longrightarrow}\left(q_{2}^{\mathcal{H}}, i\right)} \operatorname{wgt}_{\mathcal{P}}(r)=\sum_{r: q_{1} \stackrel{n w}{\longrightarrow} q_{2}} \operatorname{wgt}_{\mathcal{A}}(r)=\sum_{r:\left(q_{1}^{\mathcal{V}}, i\right) \stackrel{\Phi \cdot(n w)}{\longrightarrow}\left(q_{2}^{\mathcal{V}}, i\right)} \operatorname{wgt}_{\mathcal{P}}(r) .
$$


This is easy to see if $\nu=\emptyset$. Let $\nu \neq \emptyset$ and let $k$ be the minimal call position and let $\ell$ be the corresponding return position. Let $n w_{1}=n w[1, k-1], n w_{2}=n w[k+1, \ell-1]$ and $n w_{3}=n w[\ell+1, n]$ (we assume that all nested words exist, the cases where they do not exist are similar). Then

$$
\begin{aligned}
& \sum_{r:\left(q_{1}^{\mathcal{H}}, i\right) \stackrel{\Phi_{\mathcal{O}}(n w)}{\longrightarrow}\left(q_{2}^{\mathcal{H}}, i\right)} \operatorname{wgt}_{\mathcal{P}}(r)=
\end{aligned}
$$

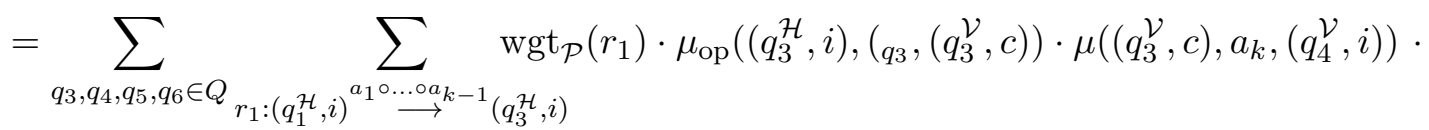

$$
\begin{aligned}
& \left.\cdot \sum_{r_{2}:\left(q_{4}^{\mathcal{V}}, i\right) \stackrel{\Phi}{\left.\stackrel{(}{\longrightarrow} w_{2}\right)}\left(q_{5}^{\mathcal{V}}, i\right)} \operatorname{wgt}_{\mathcal{P}}\left(r_{2}\right) \cdot \mu\left(\left(q_{5}^{\mathcal{V}}, i\right), a_{\ell},\left(q_{5}^{\mathcal{V}}, a_{\ell}\right)\right) \cdot \mu_{\mathrm{cl}}\left(\left(q_{5}^{\mathcal{V}}, a_{\ell}\right),\right)_{q_{3}},\left(q_{6}^{\mathcal{H}}, i\right)\right) \cdot \\
& \sum_{r_{3}:\left(q_{6}^{\mathcal{H}}, i\right) \stackrel{\Phi \circ\left(n w_{3}\right)}{\longrightarrow}\left(q_{2}^{\mathcal{H}}, i\right)} \operatorname{wgt}_{\mathcal{P}}\left(r_{3}\right) \\
& =\sum_{q_{3}, q_{4}, q_{5}, q_{6} \in Q} \sum_{r_{1}: q_{1} \stackrel{n w_{1}}{\rightarrow} q_{3}} \operatorname{wgt}_{\mathcal{A}}\left(r_{1}\right) \cdot \delta_{\text {call }}\left(q_{3}, a_{k}, q_{4}\right) \cdot \sum_{r_{2}: q_{4} \stackrel{n w_{2}}{\rightarrow} q_{5}} \operatorname{wgt}_{\mathcal{A}}\left(r_{2}\right) \cdot \delta_{\text {ret }}\left(q_{5}, q_{3}, a_{\ell}, q_{6}\right) \cdot \\
& \cdot \sum_{r_{3}: q_{6} \stackrel{n w_{3}}{\longrightarrow} q_{2}} \operatorname{wgt}_{\mathcal{A}}\left(r_{3}\right) \\
& =\sum_{r: q_{1} \stackrel{n w}{\longrightarrow} q_{2}} \operatorname{wgt}_{\mathcal{A}}(r)
\end{aligned}
$$

We can proceed analogously for $\Phi_{\bullet}$. Now the result follows from the definition of $\lambda$ and $\gamma$.

We can now prove Theorem 3.6

Proof of Theorem 3.6. We prove Theorem 3.6(a). Let $S: \mathrm{NW}(\Delta) \rightarrow \mathbb{K}$ be regular. By Proposition [5.8, $\Phi_{\circ}(S): \operatorname{TXT}(\Delta) \rightarrow \mathbb{K}$ is regular and hence sREMSO(K)-definable by Theorem 4.2. Now we get that $\Phi_{\circ}^{-1}\left(\Phi_{\circ}(S)\right)=S$ is $\operatorname{sREMSO}(\mathbb{K})$-definable by Proposition 5.2 and Corollary 5.4.

Conversely, let $S: \mathrm{NW}(\Delta) \rightarrow \mathbb{K}$ be $\operatorname{sRMSO}(\mathbb{K})$-definable. By Corollary 5.6 and Proposition 5.2, $\Phi_{\circ}(S): \operatorname{TXT}(\Delta) \rightarrow \mathbb{K}$ is $\operatorname{sRMSO}(\mathbb{K})$-definable and thus by Theorem 4.2 regular. From Proposition 5.7 we conclude that $\Phi_{\circ}^{-1}\left(\Phi_{\circ}(S)\right)=S$ is regular, too.

Similarly we get Theorem 3.6(b) from Theorem 4.2(b). Theorem 3.6(c) follows from Theorem 4.2(c).

Again note that all proofs are constructive. Hence, given a sentence $\varphi$ in $\operatorname{sRMSO}(\mathbb{K})$ (resp. swRMSO $(\mathbb{K}), \operatorname{MSO}(\mathbb{K})$ ) we can effectively construct a WNWA $\mathcal{A}$ such that $\|\mathcal{A}\|=\llbracket \varphi \rrbracket$. Conversely, given a WNWA $\mathcal{A}$ we can construct an $\operatorname{sREMSO}(\mathbb{K})$ sentence $\varphi$ such that $\|\mathcal{A}\|=\llbracket \varphi \rrbracket$. The following results follow now easily form the corresponding results for series over alternating texts [30].

Corollary 5.9. Let $\mathbb{K}$ be a locally finite semiring or let $\mathbb{K}$ be a ring and let $S: \mathrm{NW}(\Delta) \rightarrow \mathbb{K}$ be regular such that $S(\mathrm{NW}(\Delta)) \subseteq \mathbb{K}$ is finite. Moreover, let $A \subseteq \mathbb{K}$. Then $S^{-1}(A)$ is regular.

Corollary 5.10. Let $\mathbb{K}$ be a computable field or a computable locally finite semiring and let $S_{1}, S_{2}: \mathrm{NW}(\Delta) \rightarrow \mathbb{K}$ be regular. It is decidable whether $S_{1}=S_{2}$. 
Corollary 5.11. Let $\mathbb{K}$ be a computable zero-sum free semiring and let $S: \mathrm{NW}(\Delta) \rightarrow \mathbb{K}$ be regular. It is decidable whether $(S, n w)=0$ for all $n w \in \mathrm{NW}(\Delta)$.

Note that one motivation of transforming formulae in automata is solving their satisfiability problem. The last two corollaries can be seen as a extension of this: We have shown that given a formula in $\varphi \in \operatorname{sRMSO}(\mathbb{K})($ resp. $\varphi \in \operatorname{swRMSO}(\mathbb{K})$, resp. $\varphi \in \operatorname{MSO}(\mathbb{K})$ ) we can effectively translate it into a weighted nested word automaton $\mathcal{A}$. Now, provided the semiring is either zero-sum free or locally finite or a field, using the last two corollaries we can test whether there is a nested word $n w$ which gets assigned a non-zero value, i.e. $(\|\mathcal{A}\|, n w)=(\llbracket \varphi \rrbracket, n w) \neq 0$.

\section{An Applichtion to Algebraic Formal Power Series}

In this section we consider algebraic formal power series and show that they arise as the projections of regular nested word series and regular alternating text series. Applying then our logical characterizations of the latter we obtain characterizations of algebraic formal power series in terms of weighted logics generalizing results of Lautemann, Schwentick and Thérien [27] on context-free languages. Algebraic formal power series have been considered initially already by Chomsky and Schützenberger [8] and have since been intensively studied by Kuich and others. Textbooks containing several aspects of algebraic formal power series are [37] and [26]. The reader is also referred to the survey articles [25] and [35.

Let $\Delta^{*}$ be the free monoid over $\Delta$ and let $\varepsilon$ denote the empty word. A formal power series is a function $S: \Delta^{*} \rightarrow \mathbb{K}$. We denote the empty word by $\varepsilon$. Given two formal power series $S_{1}, S_{2}$, their Cauchy product, denoted $S_{1} \cdot S_{2}$ or $S_{1} S_{2}$, is given by $\left(S_{1} \cdot S_{2}, w\right)=$ $\sum_{w_{1} w_{2}=w}\left(S_{1}, w_{1}\right)\left(S_{2}, w_{2}\right)$ for all $w \in \Delta^{*}$. By $S_{1} \odot S_{2}$ we denote the pointwise product also called the Hadamard product and by $S_{1}+S_{2}$ their pointwise sum. Moreover, if $k \in \mathbb{K}$, then the formal power series $k . S$ is given by $(k . S, w)=k \cdot(S, w)$ for all $w \in \Delta^{*}$. Let $\mathbb{1}_{L}$ denote the characteristic series of a language $L \subseteq \Delta^{*}$. We identify $w$ and $\mathbb{1}_{\{w\}}$. Let $\mathcal{X}$ be an alphabet of variables such that $\Delta \cap \mathcal{X}=\emptyset$. A polynomial $P$ over $(\Delta \cup \mathcal{X})$ is a mapping $P:(\Delta \cup \mathcal{X})^{*} \rightarrow \mathbb{K}$ such that its support is finite, i.e. the $\operatorname{set} \operatorname{supp}(P)=\left\{w \in(\Delta \cup \mathcal{X})^{*} \mid(P, w) \neq 0\right\}$ is finite.

Definition 6.1. A collection of polynomials $\left(P_{X}\right)_{X \in \mathcal{X}}$ over $(\Delta \cup \mathcal{X})$ is called an algebraic system with variables in $\mathcal{X}$.

The supports of the polynomials $P_{X}$ in the last definition are thus finite sets consisting of words of the form $u_{1} X_{1} \ldots u_{k} X_{k} u_{k+1}$ where $u_{j} \in \Delta^{*}$ and $X_{j} \in \mathcal{X}$. We say that a collection $\left(S_{X}\right)_{X \in \mathcal{X}}$ of formal power series $S_{X}: \Delta^{*} \rightarrow \mathbb{K}$ is a solution of the algebraic system $\left(P_{X}\right)_{X \in \mathcal{X}}$ if for all $X \in \mathcal{X}$,

$$
S_{X}=\sum_{u_{1} X_{1} \ldots u_{k} X_{k} u_{k+1} \in \operatorname{supp}\left(P_{X}\right)}\left(P_{X}, u_{1} X_{1} \ldots u_{k} X_{k} u_{k+1}\right) \cdot u_{1} S_{X_{1}} \cdots u_{k} S_{X_{k}} u_{k+1} .
$$

An algebraic system $\left(P_{X}\right)_{X \in \mathcal{X}}$ is proper if $\left(P_{X}, Y\right)=\left(P_{X}, \varepsilon\right)=0$ for all $X, Y \in \mathcal{X}$. A formal power series $S$ having the property that $(S, \varepsilon)=0$ is called quasiregular. A proper algebraic system has a unique quasiregular solution [37], more precisely a proper algebraic system has exactly one solution $\left(S_{X}\right)_{X \in \mathcal{X}}$ such that $\left(S_{X}, \varepsilon\right)=0$ for all $X \in \mathcal{X}$.

Definition 6.2. A formal power series $S: \Delta^{*} \rightarrow \mathbb{K}$ is an algebraic formal power series if it is a component of the quasiregular solution of a proper algebraic system. 2

\footnotetext{
${ }^{2}$ This definition is given in [37]. In [25, 26] a series $S$ is called algebraic if its quasiregular part $\mathbb{1}_{\Delta+} \odot S$ is the component of the quasiregular solution of a proper algebraic system.
} 
We note that over the 2-valued Boolean algebra $\mathbb{B}$ these series correspond exactly to the $\varepsilon$-free context-free languages. The bijection is given by supp.

To warm up let us discuss some easy manipulations of algebraic systems. For this, let us consider some algebraic system $\left(P_{X}\right)_{X \in \mathcal{X}}$. Let $X, Y \in \mathcal{X}$. Clearly, it follows directly from the definition of a solution that we can substitute an occurrence of $Y$ in some word of the support of $P_{X}$ by $P_{Y}$ without altering the solutions of the system. More formally: Let $u Y v \in \operatorname{supp}\left(P_{X}\right)$. Let $\left(P_{X}^{\prime}\right)_{X \in \mathcal{X}}$ be given from $\left(P_{X}\right)_{X \in \mathcal{X}}$ by replacing $P_{X}$ with the polynomial

$$
\left(\mathbb{1}_{\operatorname{supp}\left(P_{X}\right) \backslash\{u Y v\}} \odot P_{X}\right)+\left(P_{X}, u Y v\right) \cdot u P_{Y} v .
$$

Then $\left(P_{X}\right)_{X \in \mathcal{X}}$ and $\left(P_{X}^{\prime}\right)_{X \in \mathcal{X}}$ are equivalent, i.e. any solution of $\left(P_{X}\right)_{X \in \mathcal{X}}$ is a solution of $\left(P_{X}^{\prime}\right)_{X \in \mathcal{X}}$ and vice versa. An algebraic system $\left(P_{X}\right)_{X \in \mathcal{X}}$ is called weakly strict, if $\operatorname{supp}\left(P_{X}\right) \subseteq\{\varepsilon\} \cup \Delta(\Delta \cup \mathcal{X})^{*}$ for all $X \in \mathcal{X}$. Let us now assume that $\left(P_{X}\right)_{X \in \mathcal{X}}$ is weakly strict. Then for any fixed $k \in \mathbb{N}$ by repeated substitution we can obtain an equivalent algebraic system $\left(P_{X}^{k}\right)_{X \in \mathcal{X}}$ such that for all $X \in \mathcal{X}$ any $w \in \operatorname{supp}\left(P_{X}^{k}\right) \backslash \Delta^{*}$ contains at least $k$ letters from $\Delta$. We conclude that any weakly strict algebraic system $\left(P_{X}\right)_{X \in \mathcal{X}}$ has a unique solution $\left(S_{X}\right)_{X \in \mathcal{X}}$ which is given by $\left(S_{X}, w\right)=\left(P_{X}^{k}, w\right)$ for all $w \in \Delta^{*}$ such that $|w|<k$.

Now, we continue by manipulating $\left(P_{X}^{k}\right)_{X \in \mathcal{X}}$. Let again $X \in \mathcal{X}$ and let $w \in \operatorname{supp}\left(P_{X}^{k}\right)$ with $|w|<k$. Let $Y \in \mathcal{X} \backslash\{X\}$. For any possible choice of occurrences of $X$ in the support of $P_{Y}$ we substitute these occurrences by $w$. More precisely, for all $Y \in \mathcal{X} \backslash\{X\}$ replace $P_{Y}^{k}$ by the polynomial

$$
\sum_{\substack{i \in \mathbb{N} \\ u_{i}, u_{i+1} \in(\Delta \cup \mathcal{X})^{*}}}\left(P_{Y}^{k}, u_{1} X u_{2} \ldots u_{i} X u_{i+1}\right) \cdot u_{1} \cdot\left(P_{X}^{k}, w\right) \cdot w \cdot u_{2} \cdots u_{i} \cdot\left(P_{X}^{k}, w\right) \cdot w \cdot u_{i+1} \cdot
$$

Furthermore, replace $P_{X}^{k}$ by the polynomial

$$
\sum_{\substack{i \in \mathbb{N} \\ \mathbb{1}_{(\Delta \cup \mathcal{X})^{*} \backslash\{w\}} \odot}}\left(P_{X}^{k}, u_{1} X u_{2} \ldots u_{i} X u_{i+1}\right) \cdot u_{1} \cdot\left(P_{X}^{k}, w\right) \cdot w \cdot u_{2} \cdots u_{i} \cdot\left(P_{X}^{k}, w\right) \cdot w \cdot u_{i+1} .
$$

Observe that these sums are in fact finite and note that in these definitions the factors $u_{1}, u_{2}, \ldots, u_{i}, u_{i+1} \in(\Delta \cup \mathcal{X})^{*}$ may contain occurrences of $X$. The resulting system is again weakly strict and has thus a unique solution $\left(S_{X}^{\prime}\right)_{X \in \mathcal{X}}$. A straightforward but cumbersome calculation, which we omit here, shows, using the distributivity of the semiring of formal power series, that $S_{Y}^{\prime}=S_{Y}$ for all $Y \in \mathcal{X} \backslash\{X\}$ and $S_{X}^{\prime}=\mathbb{1}_{(\Delta \cup \mathcal{X})^{*} \backslash\{w\}} \odot S_{X}$. For fixed $0 \leq k^{\prime}<k$ by repeated application we can thus obtain a proper and weakly strict algebraic system $\left(R_{X}\right)_{X \in \mathcal{X}}$ such that the quasiregular and unique solution $\left(T_{X}\right)_{X \in \mathcal{X}}$ is given by $\left(\mathbb{1}_{\left\{w\left|k^{\prime}<\right| w \mid\right\}} \odot S_{X}\right)_{X \in \mathcal{X}}$. In particular, it follows that the quasiregular part $\mathbb{1}_{\Delta^{+}} \odot S_{X}$ of $S_{X}$ is algebraic for any $X \in \mathcal{X}$.

6.1. Nested Word Series and Their Projections. Next, we consider the projections of regular nested word series and show that they give rise exactly to the algebraic series. The projection $\pi(n w)$ of a nested word $n w=(w, \nu) \in \mathrm{NW}(\Delta)$ is simply the word $w$, 
i.e. we forget the nesting relation. This projection is canonically generalized to languages $L \subseteq \mathrm{NW}(\Delta)$ by setting $\pi(L)=\{\pi(n w) \mid n w \in L\}$ and to series $S: \mathrm{NW}(\Delta) \rightarrow \mathbb{K}$ by letting

$$
\begin{aligned}
\pi(S): \Delta^{*} & \rightarrow \mathbb{K} \\
w & \mapsto \sum_{\substack{n w \in \mathrm{NW}(\Delta) \\
w=\pi(n w)}}(S, n w) .
\end{aligned}
$$

Proposition 6.3. Let $S: \mathrm{NW}(\Delta) \rightarrow \mathbb{K}$ be regular. Then $\pi(S): \Delta^{*} \rightarrow \mathbb{K}$ is an algebraic formal power series.

Proof. Let $\mathcal{A}=(Q, \iota, \delta, \kappa)$ be a WNWA such that $\|\mathcal{A}\|=S$. We define a weakly strict algebraic system $\left(P_{\left(q_{1}, q_{2}\right)}\right)_{q_{1}, q_{2} \in Q}$ with variables in $Q^{2}$ such that for its solution $\left(S_{\left(q_{1}, q_{2}\right)}\right)_{q_{1}, q_{2} \in Q}$ we have for all $w \in \Delta^{*}$ with $|w| \geq 1$ :

$$
\left(S_{\left(q_{1}, q_{2}\right)}, w\right)=\sum_{\substack{n w \in \mathrm{NW}(\Delta) \\ \pi(n w)=w}} \sum_{r: q_{1} \stackrel{n w}{\rightarrow} q_{2}} \operatorname{wgt}_{\mathcal{A}}(r) .
$$

The idea is to simulate the transitions of a weighted nested word automaton. For this we will partition the set of nested words of length at least two in three different classes. First the class of nested words where the first and the last position are either corresponding call and return positions or both internal positions. The second class consists of nested words where either the first position is a call position and the last position is an internal position or the last position is a return position and the first position is an internal position. And the last class consists of any other, i.e. where the first position is a call position and the last position is a return position which do not correspond to each other. Using this partition we define for all $q_{1}, q_{2} \in Q$ the polynomial $P_{\left(q_{1}, q_{2}\right)}:\left(\Delta \cup Q^{2}\right)^{*} \rightarrow \mathbb{K}$ as follows:

$$
\begin{aligned}
& \left(P_{\left(q_{1}, q_{2}\right)}, w\right)= \\
& \left\{\begin{array}{lc}
1 & \text { if } q_{1}=q_{2} \text { and } w=\varepsilon \\
\delta_{\text {int }}\left(q_{1}, a, q_{2}\right) & \text { if } w=a \text { for some } a \in \Delta \\
\delta_{\text {int }}\left(q_{1}, a, q_{3}\right) \cdot \delta_{\text {int }}\left(q_{4}, b, q_{2}\right)+ & \text { if } w=a\left(q_{3}, q_{4}\right) b \\
\delta_{\text {call }}\left(q_{1}, a, q_{3}\right) \cdot \delta_{\text {ret }}\left(q_{4}, q_{1}, b, q_{2}\right) & \text { for some } a, b \in \Delta, q_{3}, q_{4} \in Q \\
\delta_{\text {call }}\left(q_{1}, a, q_{3}\right) \cdot \delta_{\text {ret }}\left(q_{4}, q_{1}, b, q_{5}\right) \cdot \delta_{\text {int }}\left(q_{6}, c, q_{2}\right)+ & \text { if } w=a\left(q_{3}, q_{4}\right) b\left(q_{5}, q_{6}\right) c \\
\delta_{\text {int }}\left(q_{1}, a, q_{3}\right) \cdot \delta_{\text {call }}\left(q_{4}, b, q_{5}\right) \cdot \delta_{\text {ret }}\left(q_{6}, q_{4}, c, q_{2}\right) & \text { for some } a, b, c \in \Delta \\
\delta_{\text {call }}\left(q_{1}, a, q_{3}\right) \cdot \delta_{\text {ret }}\left(q_{4}, q_{1}, b, q_{5}\right) \cdot & \text { and } q_{3}, q_{4}, q_{5}, q_{6} \in Q \\
\delta_{\text {call }}\left(q_{6}, c, q_{7}\right) \cdot \delta_{\text {ret }}\left(q_{8}, q_{6}, d, q_{2}\right) & \text { if } w=a\left(q_{3}, q_{4}\right) b\left(q_{5}, q_{6}\right) c\left(q_{7}, q_{8}\right) d \\
& \text { for some } a, b, c, d \in \Delta \\
0 & \text { and } q_{3}, q_{4}, q_{5}, q_{6}, q_{7}, q_{8} \in Q
\end{array}\right.
\end{aligned}
$$

This is a weakly strict algebraic system having a necessarily unique solution $\left(S_{\left(q_{1}, q_{2}\right)}\right)_{q_{1}, q_{2} \in Q}$. We show by induction on the length of $w$ that (6.1) holds. For $|w|=1$ this is easy to see. Now let $|w|>1$. Then 


$$
\begin{aligned}
& \left(S_{\left(q_{1}, q_{2}\right)}, w\right)= \\
& =\sum_{q_{3}, q_{4}} \in Q\left[\delta_{\text {int }}\left(q_{1}, a_{1}, q_{3}\right) \cdot \delta_{\text {int }}\left(q_{4}, a_{n}, q_{2}\right)+\delta_{\text {call }}\left(q_{1}, a_{1}, q_{3}\right) \cdot \delta_{\text {ret }}\left(q_{4}, q_{1}, a_{n}, q_{2}\right)\right] \\
& \cdot\left(S_{\left(q_{3}, q_{4}\right)}, a_{2} \ldots a_{n-1}\right)+ \\
& +\sum_{2 \leq i \leq n-1} \sum_{q_{3}, q_{4}, q_{5}, q_{6} \in Q}\left[\delta_{\text {call }}\left(q_{1}, a_{1}, q_{3}\right) \cdot \delta_{\text {ret }}\left(q_{4}, q_{1}, a_{i}, q_{5}\right) \cdot \delta_{\text {int }}\left(q_{6}, a_{n}, q_{2}\right)+\right. \\
& \left.+\delta_{\mathrm{int}}\left(q_{1}, a_{1}, q_{3}\right) \cdot \delta_{\mathrm{call}}\left(q_{4}, a_{i}, q_{5}\right) \cdot \delta_{\mathrm{ret}}\left(q_{6}, q_{4}, a_{n}, q_{2}\right)\right] \\
& \cdot\left(S_{\left(q_{3}, q_{4}\right)}, a_{2} \ldots a_{i-1}\right) \cdot\left(S_{\left(q_{5}, q_{6}\right)}, a_{i+1} \ldots a_{n-1}\right) \\
& +\sum_{2 \leq i<j \leq n-1} \sum_{\substack{q_{3}, q_{4}, q_{5}, q_{6}, q_{7}, q_{8} \in Q}} \delta_{\text {call }}\left(q_{1}, a_{1}, q_{3}\right) \cdot \delta_{\text {ret }}\left(q_{4}, q_{1}, a_{i}, q_{5}\right) \cdot \delta_{\text {call }}\left(q_{6}, a_{j}, q_{7}\right) \cdot \delta_{\text {ret }}\left(q_{8}, q_{6}, a_{n}, q_{2}\right) \\
& \cdot\left(S_{\left(q_{3}, q_{4}\right)}, a_{2} \ldots a_{i-1}\right) \cdot\left(S_{\left(q_{5}, q_{6}\right)}, a_{i+1} \ldots a_{j-1}\right) \cdot\left(S_{\left(q_{7}, q_{8}\right)}, a_{j+1} \ldots a_{n-1}\right)
\end{aligned}
$$

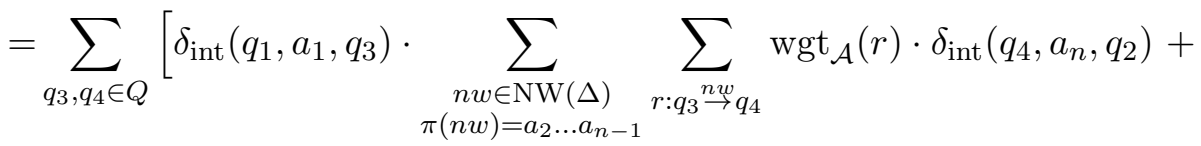

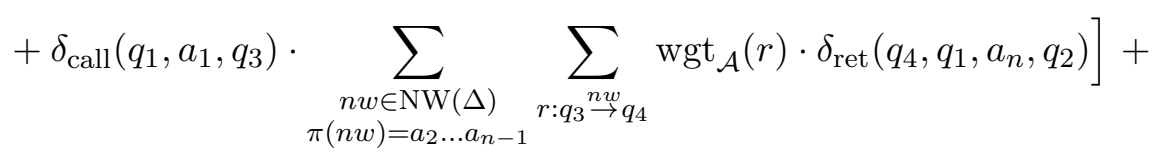

$$
\begin{aligned}
& +\sum_{2 \leq i \leq n-1} \sum_{\substack{q_{3}, q_{4} \\
q_{5}, q_{6} \in Q}}\left[\delta_{\text {call }}\left(q_{1}, a_{1}, q_{3}\right) \cdot \sum_{\substack{n w_{1} \in \mathrm{NW}(\Delta) \\
\pi\left(n w_{1}\right)=a_{2} \ldots a_{i-1}}} \sum_{\substack{r_{1}: q_{3} \stackrel{n w_{1}}{\rightarrow} q_{4} \\
\operatorname{wgt}_{\mathcal{A}}}}\left(r_{1}\right) \cdot \delta_{\text {ret }}\left(q_{4}, q_{1}, a_{i}, q_{5}\right) .\right. \\
& \sum_{\substack{n w_{2} \in \mathrm{NW}(\Delta) \\
\pi\left(n w_{2}\right)=a_{i+1} \ldots a_{n-1}}} \sum_{\substack{n w_{2} \\
r_{2}: q_{5} q_{6}}} \operatorname{wgt}_{\mathcal{A}}\left(r_{2}\right) \cdot \delta_{\text {int }}\left(q_{6}, a_{n}, q_{2}\right)+ \\
& +\delta_{\text {int }}\left(q_{1}, a_{1}, q_{3}\right) \cdot \sum_{\substack{n w_{1} \in \mathrm{NW}(\Delta) \\
\pi\left(n w_{1}\right)=a_{2} \ldots a_{i-1}}} \sum_{\substack{n w_{1} \\
r_{1}: q_{3} \stackrel{\rightarrow}{\rightarrow} q_{4}}} \operatorname{wgt}_{\mathcal{A}}\left(r_{1}\right) \cdot
\end{aligned}
$$

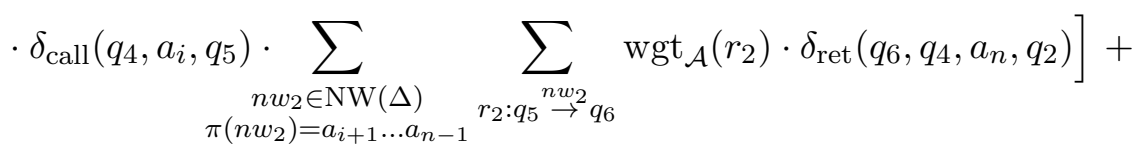

$$
\begin{aligned}
& +\sum_{2 \leq i<j \leq n-1} \sum_{\substack{q_{3}, q_{4}, q_{5}, q_{6}, q_{7}, q_{8} \in Q}} \delta_{\text {call }}\left(q_{1}, a_{1}, q_{3}\right) \cdot \sum_{\substack{n w_{1} \in \mathrm{NW}(\Delta) \\
\pi\left(n w_{1}\right)=a_{2} \ldots a_{i-1}}} \sum_{\substack{r_{1}: q_{3} \rightarrow w_{1} \rightarrow q_{4} \\
q^{\prime}}} \operatorname{wgt}_{\mathcal{A}}\left(r_{1}\right) \cdot \delta_{\text {ret }}\left(q_{4}, q_{1}, a_{i}, q_{5}\right) \cdot
\end{aligned}
$$

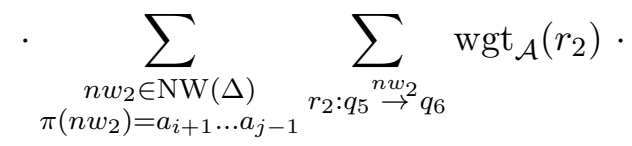

$$
\begin{aligned}
& \cdot \delta_{\text {call }}\left(q_{6}, a_{j}, q_{7}\right) \cdot \sum_{\substack{n w_{3} \in \mathrm{NW}(\Delta) \\
\pi\left(n w_{3}\right)=a_{j+1} \ldots a_{n-1}}} \sum_{r_{3}: q_{7} \stackrel{n w_{3}}{\rightarrow} q_{8}} \operatorname{wgt}_{\mathcal{A}}\left(r_{3}\right) \cdot \delta_{\mathrm{ret}}\left(q_{8}, q_{6}, a_{n}, q_{2}\right) \\
& =\sum_{\pi(n w)=w} \sum_{r: q_{1} \rightarrow q_{2}} \operatorname{wgt}_{\mathcal{A}}(r) .
\end{aligned}
$$


Now, let $X$ be a fresh variable and extend $\left(P_{\left(q_{1}, q_{2}\right)}\right)_{q_{1}, q_{2} \in Q}$ by adding the new polynomial $P_{X}=\sum_{q_{1}, q_{2} \in Q} \iota\left(q_{1}\right) \cdot \kappa\left(q_{2}\right) \cdot P_{\left(q_{1}, q_{2}\right)}$. Clearly, the unique solution of this extended system is obtained by adding $S_{X}=\sum_{q_{1}, q_{2} \in Q} \iota\left(q_{1}\right) \cdot \kappa\left(q_{2}\right) . S_{\left(q_{1}, q_{2}\right)}$ to $\left(S_{\left(q_{1}, q_{2}\right)}\right)_{q_{1}, q_{2} \in Q}$. The quasiregular part of $S_{X}$ equals $\pi(\|\mathcal{A}\|)$ which is thus algebraic by our considerations after Definition 6.2.

Given an algebraic system $\left(P_{X}\right)_{X \in \mathcal{X}}$ over $(\Delta \cup \mathcal{X})$ and some $X \in \mathcal{X}$, we define the underlying grammar $G_{X}=(\Delta, \mathcal{X}, X, F)$ where the set $F \subseteq \mathcal{X} \times(\mathcal{X} \cup \Delta)^{*}$ of productions is given by letting $(Y, w) \in F$ iff $\left(P_{Y}, w\right) \neq 0$. Let $u \in \Delta^{*}$. A derivation tree of $u$ under $G_{X}$ is a finite tree $t$ such that the following holds:

(a) The root is labeled with $(X, w)$ for some $w \in \operatorname{supp}\left(P_{X}\right)$.

(b) For each inner node $v$ with label $(Y, w)$ the first component of the labels of the children of $v$ from left to right yield $w$.

(c) The labels of the leaves from left to right yield $u$.

We collect all derivation trees $t$ of $u$ under $G_{X}$ in $\operatorname{Der}\left(G_{X}, u\right)$. Clearly, if $\left(P_{X}\right)_{X \in \mathcal{X}}$ is proper, then each inner node of $t$ either has a single leaf attached or branches at least binarily. Hence, in this case $\operatorname{Der}\left(G_{X}, u\right)$ is a finite set. Let $v$ be a node of $t$. If $v$ is an inner node and $(Y, w)$ its label, then we let $\operatorname{wgt}(t, v)=\left(P_{Y}, w\right)$. If $v$ is a leaf, we let $\operatorname{wgt}(t, v)=1$. Now we define the weight $\operatorname{wgt}(t)$ of $t$ by $\operatorname{wgt}(t)=\prod_{v \text { node of } t} \operatorname{wgt}(t, v)$. The following lemma seems to belong to what is sometimes called folklore, it can easily be shown by induction on the length of $w$. A proof of a similar but weaker result can be found in [37, Theorem IV.1.5].

Lemma 6.4. Let $\left(P_{X}\right)_{X \in \mathcal{X}}$ be a proper algebraic system and let $\left(S_{X}\right)_{X \in \mathcal{X}}$ be its unique quasiregular solution. Then

$$
\left(S_{X}, w\right)=\sum_{t \in \operatorname{Der}\left(G_{X}, w\right)} \operatorname{wgt}(t) \text { for all } X \in \mathcal{X} \text { and } w \in \Delta^{*} .
$$

We now show the converse of Proposition 6.3.

Proposition 6.5. Let $R: \Delta^{*} \rightarrow \mathbb{K}$ be an algebraic formal power series. Then there is a regular nested word series $S: \mathrm{NW}(\Delta) \rightarrow \mathbb{K}$ such that $\pi(S)=R$.

Proof. Let $\left(P_{X}\right)_{X \in \mathcal{X}}$ be a proper algebraic system with quasiregular solution $\left(S_{X}\right)_{X \in \mathcal{X}}$ and let $Y \in \mathcal{X}$ such that $R=S_{Y}$. We construct a WNWA $\mathcal{A}=(Q, \iota, \delta, \kappa)$ such that $\pi(\|\mathcal{A}\|)=S_{Y}$. Any element in the support of some $P_{X}$ will define a transition in the automaton. In order not to produce $\varepsilon$-transitions, we require that each word in the support of some $P_{X}$ contains an element of $\Delta$, and in order to produce at most one call for each transition, each word in the support of some $P_{Y}$ contains at most two elements of $\mathcal{X}$. Therefore we assume the algebraic system $\left(P_{X}\right)_{X \in \mathcal{X}}$ to be in Greibach normal form [26], i.e. we require that $\operatorname{supp}\left(P_{X}\right) \subseteq \Delta \cup \Delta \mathcal{X} \cup \Delta \mathcal{X} \mathcal{X}$ for all $X \in \mathcal{X}$. Elements of $\Delta \mathcal{X} \mathcal{X}$ produce call transitions, elements in $\Delta \mathcal{X}$ produce internal transitions and elements in $\Delta$ produce return transitions. More precisely, let $Q=(\mathcal{X} \cup\{\perp\}) \times(\mathcal{X} \cup\{\perp\})$ for some fresh symbol $\perp$, and for all $X_{1}, X_{3}, X_{4} \in \mathcal{X}$ and $X_{2} \in \mathcal{X} \cup\{\perp\}$ let

$$
\begin{aligned}
\delta_{\text {call }}\left(\left(X_{1}, X_{4}\right), a,\left(X_{3}, X_{2}\right)\right) & =\left(P_{X_{1}}, a X_{3} X_{4}\right) \\
\delta_{\text {int }}\left(\left(X_{1}, X_{2}\right), a,\left(X_{3}, X_{2}\right)\right) & =\left(P_{X_{1}}, a X_{3}\right) \\
\delta_{\text {ret }}\left(\left(X_{1}, X_{2}\right),\left(X_{3}, X_{4}\right), a,\left(X_{4}, X_{2}\right)\right) & =\left(P_{X_{1}}, a\right) .
\end{aligned}
$$


Moreover, let $\delta_{\text {int }}\left(\left(X_{1}, \perp\right), a,(\perp, \perp)\right)=\left(P_{X_{1}}, a\right)$. Any other transition gets weight 0 . Furthermore, for all $X, Z \in \mathcal{X} \cup\{\perp\}$ we let

$$
\iota(X, Z)=\left\{\begin{array}{ll}
1 & \text { if } X=Y \\
0 & \text { otherwise }
\end{array} \quad \kappa(X, Z)= \begin{cases}1 & \text { if } X=Z=\perp \\
0 & \text { otherwise }\end{cases}\right.
$$

The idea is to simulate a derivation tree of the underlying grammar $G_{Y}$ traversed from the left to the right. More precisely, when processing a production $\left(X_{1}, a X_{3} X_{2}\right)$ in a derivation tree, then a call transition is executed and we continue in a state with first component $X_{3}$. At the return position the automaton changes to $X_{2}$. Since the automaton looks back to the state in which the automaton was before the corresponding call position, it has to guess $X_{2}$ in advance. This is stored in the second component which was introduced for this reason. One can show by induction on $|w|$ that for all $w \in \Delta^{*}, X \in \mathcal{X}$ and $Z^{\prime} \in \mathcal{X} \cup\{\perp\}$ we have

$$
\left(S_{X}, w a\right)=\sum_{\nu \in \operatorname{Nest}_{|w|}} \sum_{\substack{X^{\prime} \in \mathcal{X}, Z \in \mathcal{X} \cup\{\perp\} \\ r:(X, Z) \stackrel{(w, \nu)}{\rightarrow}\left(X^{\prime}, Z^{\prime}\right)}} \operatorname{wgt}_{\mathcal{A}}(r) \cdot\left(P_{X^{\prime}}, a\right)
$$

where we make the convention that there is a run $r:(X, Z) \stackrel{(\varepsilon, \emptyset)}{\rightarrow}\left(X^{\prime}, Z^{\prime}\right)$ iff $X=X^{\prime}$ and $Z=Z^{\prime}$. Moreover, for this run we let $\operatorname{wgt}_{\mathcal{A}}(r)=1$. Now the result follows easily from the observation that by the definition of $\delta$ the last transition of a run $r:(Y, Z) \stackrel{(w, \nu)}{\rightarrow}(\perp, \perp)$ with $\operatorname{wgt}(r) \neq 0$ must be an internal transition.

Subsequently we make use of the following well known result [26]. We just indicate how it can be obtained in this context using Propositions 6.3 and 6.5. but note that a more elementary proof and more general results can be found in [26, Chapter 15].

Corollary 6.6 (Kuich \& Salomaa [26, Lemma 15.2]). Let $S: \Delta^{*} \rightarrow \mathbb{K}$ be an algebraic formal power series. Then there is an algebraic system $\left(P_{X}\right)_{X \in \mathcal{X}}$ such that $\operatorname{supp}\left(P_{X}\right) \subseteq$ $\Delta \cup \Delta(\Delta \cup \mathcal{X})^{*} \Delta$ for all $X \in \mathcal{X}$ and $S=S_{X}$ for some $X \in \mathcal{X}$.

Proof. By Proposition 6.5, $S$ is the projection of some regular nested word series $R$ : $\operatorname{NW}(\Delta) \rightarrow \mathbb{K}$. Now let $\mathcal{A}$ be a WNWA and $Q$ its set of states such that $\|\mathcal{A}\|=R$. Consider the weakly strict algebraic system $\left(P_{X},\left(P_{\left(q_{1}, q_{2}\right)}\right)_{q_{1}, q_{2} \in Q}\right)$ of the proof of Proposition 6.3 and its unique solution $\left(S_{X},\left(S_{\left(q_{1}, q_{2}\right)}\right)_{q_{1}, q_{2} \in Q}\right)$. Using the manipulations given after Definition 6.2 we can transform this system into a system of the required form having as a solution the quasiregular part of $S_{X}$ which equals $S$.

6.2. A Logical Characterization of Algebraic Formal Power Series. Our aim is to give a logical characterization of algebraic formal power series in the spirit of Lautemann, Schwentick and Thérien [27]. They showed that the context-free languages are precisely the languages which can be defined by second-order sentences over words of the form $\exists \nu . \varphi$ where $\varphi$ is a first-order formula and $\nu$ a binary predicate ranging over nesting relations 3 . We identify a word $a_{1} \ldots a_{n} \in \Delta^{*}$ with the structure $([n], \leq, \lambda)$, where $\leq$ is the canonical order of $[n]$ and $\lambda:[n] \rightarrow \Delta$ is given by $\lambda(i)=a_{i}$ for all $i \in[n]$. Let $\varphi$ be a weighted second-order formula over words containing, apart from a single 2-ary relation variable $\nu$,

\footnotetext{
${ }^{3}$ In [27] nesting relations were named matchings.
} 
only 1-ary relation variables. In other words let $\varphi \in \operatorname{MSO}(\mathbb{K}, \Delta, \leq, \nu)$. Let $\operatorname{Free}(\varphi) \subseteq \mathcal{V}$, $w \in \Delta^{*}$ and $\gamma$ a $(\mathcal{V}, w)$-assignment. We define the semantics $\llbracket \exists \nu . \varphi \rrbracket^{\text {nest }}: \Delta^{*} \rightarrow \mathbb{K}$ by letting

$$
\left(\llbracket \exists \nu \cdot \varphi \rrbracket^{\text {nest }},(w, \gamma)\right)=\sum_{\nu \in \mathrm{Nest}_{|w|}}(\llbracket \varphi \rrbracket,((w, \nu), \gamma)) .
$$

Using our characterization of nested word automata by means of weighted logics (Theorem [3.6), we may reformulate Proposition 6.3 as follows:

Corollary 6.7. Let $\varphi \in \operatorname{sRMSO}(\mathbb{K}, \Delta, \leq, \nu)$ be a sentence. Then $\llbracket \exists \nu . \varphi \rrbracket^{n e s t}: \Delta^{*} \rightarrow \mathbb{K}$ is an algebraic formal power series.

Next we show a result which sharpens Proposition 6.5. For this we follow the proof of Lautemann, Schwentick and Thérien [27, Theorem 2.1] with small changes in the details.

Proposition 6.8. Let $S$ be an algebraic formal power series. Then there is a sentence $\varphi \in \operatorname{sRFO}(\mathbb{K}, \Delta, \leq, \nu)$ such that $S=\llbracket \exists \nu . \varphi \rrbracket^{\text {nest }}$.

Proof. We use an idea of Lautemann, Schwentick and Thérien [27] and adapt it to the weighted setting. This requires that we have to be more careful in order not to count weights twice.

A normal form. By Corollary 6.6 we may assume that $S$ is the component of the solution of an algebraic system with variables in $\mathcal{X}$ having all supports in $\Delta \cup \Delta(\Delta \cup \mathcal{X})^{*} \Delta$. By the transformations discussed after Definition 6.2 we obtain from this a proper algebraic system $\left(P_{X}^{\prime}\right)_{X \in \mathcal{X}}$ with solution $\left(S_{X}\right)_{X \in \mathcal{X}}$ such that for all $X \in \mathcal{X}, \operatorname{supp}\left(P_{X}^{\prime}\right)$ does not contain elements of $\Delta \cup\{\varepsilon\}$ and $\mathbb{1}_{\left\{w \in \Delta^{*}|| w \mid>1\right\}} \odot S$ is a component of the solution. Clearly, it suffices to show the proposition for the latter series instead of $S$.

Now we proceed as in [27] and transform the system $\left(P_{X}^{\prime}\right)_{X \in \mathcal{X}}$ into an equivalent system $\left(P_{X}\right)_{X \in \mathcal{X}}$. Let $w \in \operatorname{supp}\left(P_{X}^{\prime}\right)$ for some $X \in \mathcal{X}$. The image of $w$ under the homomorphism which is the identity on $\Delta$ and maps any $Y \in \mathcal{X}$ to the fresh symbol $\mid$ is called the pattern $\operatorname{patt}(w)$ of $w$. Let us now fix a strict linear order $<$ on $\mathcal{X}$. Similarly to [27, we proceed along this linear order. Let $X$ be the current symbol. In order to obtain $P_{X}$ we substitute iteratively some $Z \in \mathcal{X}$ in some $w \in \operatorname{supp}\left(P_{X}^{\prime}\right)$ by $P_{Z}^{\prime}$ (cf. considerations after Definition 6.2) until for all $Y \in \mathcal{X}$, with $Y<X$, $\operatorname{patt}(w) \neq \operatorname{patt}\left(w^{\prime}\right)$ for all $w^{\prime} \in \operatorname{supp}\left(P_{Y}\right) \backslash \Delta^{*}$ and $w \in \operatorname{supp}\left(P_{X}\right) \backslash \Delta^{*}$. This is possible since by our considerations after Definition 6.2 we can ensure that all elements in $\operatorname{supp}\left(P_{X}\right) \backslash \Delta^{*}$ are longer than all elements in $\operatorname{supp}\left(P_{Y}\right) \backslash \Delta^{*}$ for all $Y<X$. We finally obtain a proper algebraic system $\left(P_{X}\right)_{X \in \mathcal{X}}$ equivalent to $\left(P_{X}^{\prime}\right)_{X \in \mathcal{X}}$ having the following properties:

(1) $\operatorname{supp}\left(P_{X}\right) \subseteq \Delta(\Delta \cup \mathcal{X})^{+} \Delta$ for all $X \in \mathcal{X}$.

(2) For all $X, Y \in \mathcal{X}$, if $\operatorname{patt}(w)=\operatorname{patt}\left(w^{\prime}\right)$ for some $w \in \operatorname{supp}\left(P_{X}\right) \backslash \Delta^{*}$ and $w^{\prime} \in$ $\operatorname{supp}\left(P_{Y}\right) \backslash \Delta^{*}$, then $X=Y$.

Let us fix $Y \in \mathcal{X}$. We now proceed by giving a sentence $\varphi_{Y} \in \operatorname{sRFO}(\mathbb{K})$ such that $\pi\left(\llbracket \varphi_{Y} \rrbracket\right)=$ $S_{Y}$. This will conclude the proof.

Some macros. Let $G_{Y}$ be the underlying grammar (see the definition after the proof of Proposition 6.3) and let $u \in \Delta^{*}$. The basic idea now is to assign to each derivation tree $t \in \operatorname{Der}\left(G_{Y}, u\right)$ a nesting relation $\nu_{t}$ of width $|u|$. This is done by letting $(i, j) \in \nu_{t}$ if there is an inner node of $t$ such that the leaves of the subtree rooted at this node are exactly the leaves between the $i$ th and the $j$ th leaf of $t$ (in lexicographic order including the $i$ th and the $j$ th leaf). Clearly, due to the special form of $\left(P_{X}\right)_{X \in \mathcal{X}}$ this binary relation is indeed a nesting relation. Let us now define some macros for nested words. Let $n w=$ 
$(u, \nu)=\left(a_{1} \ldots a_{k}, \nu\right) \in \mathrm{NW}(\Delta)$. Then let $\min (x)$ and $\max (y)$ express that $x$ is assigned the first position and $y$ the last position. Furthermore, the formula inchild $(x, y)$ express that $(x, y) \in \nu$ corresponds to an inner node of $t$ which has an inner node as a child.

$$
\operatorname{inchild}(x, y)=\nu(x, y) \wedge \exists z, z^{\prime} .(x<z<y) \wedge \nu\left(z, z^{\prime}\right)
$$

The macro $\operatorname{surf}\left(x, y, x_{1}, y_{1}\right)$ says that $\left(x_{1}, y_{1}\right)$ is a surface arch of $n w[x, y]$ :

$$
\begin{aligned}
\operatorname{surf}\left(x, y, x_{1}, y_{1}\right)=\left(x<x_{1}<y_{1}<y\right) & \wedge \nu\left(x_{1}, y_{1}\right) \wedge \\
& \wedge \forall z, z^{\prime} .\left(x<z<x_{1}<y_{1}<z^{\prime}<y\right) \rightarrow \neg \nu\left(z, z^{\prime}\right) .
\end{aligned}
$$

As in [27, for $v \in \Delta^{*}$ let $\psi_{v}(x, y)$ be a first-order formula that expresses there is no call strictly between positions $x$ and $y$ and that the substring given by the positions strictly between position $x$ and $y$ equals $v$. For a word $w=a v b \in \Delta^{+}$define $\vartheta_{w}(x, y)$ as follows.

$$
\vartheta_{w}(x, y)=\operatorname{Lab}_{a}(x) \wedge \operatorname{Lab}_{b}(y) \wedge \psi_{v}(x, y)
$$

Now we will need the notion of a pattern also for nested words [27]. Let $\left(i_{1}, j_{1}\right), \ldots,\left(i_{s}, j_{s}\right)$ be the sequence of all surface arches of $n w$. The pattern patt $(n w)$ of $n w$ is the string $a_{1} \ldots a_{i_{1}-1}\left|a_{j_{1}+1} \ldots a_{i_{s}-1}\right| a_{j_{s}+1} \ldots a_{k}$. Now, let $X \in \mathcal{X}$, let $w=a v_{0} X_{1} v_{1} \ldots v_{s-1} X_{s} v_{s} b \in$ $\operatorname{supp}\left(P_{X}\right) \backslash \Delta^{+}$and let $p=\operatorname{patt}(w)=a v_{0}\left|v_{1} \ldots v_{s-1}\right| v_{s} b$. We define the formula $\chi_{p}(x)$ (cf. [27]) which states that $x$ is a call position with return position $y$ and $\operatorname{patt}(n w[x, y])=p$.

$$
\begin{aligned}
& \chi_{p}(x)=\exists y \cdot \nu(x, y) \wedge \operatorname{Lab}_{a}(x) \wedge \operatorname{Lab}_{b}(y) \wedge \\
& \wedge \exists x_{1}, y_{1}, \ldots, x_{s}, y_{s} \cdot\left[\left(x<x_{1}<y_{1} \ldots<y_{s}<y\right) \wedge \psi_{v_{0}}\left(x, x_{1}\right) \wedge \ldots \wedge \psi_{v_{s}}\left(y_{s}, y\right) \wedge\right. \\
& \wedge\left(\operatorname{surf}\left(x, y, x_{1}, y_{1}\right) \wedge \ldots \wedge \operatorname{surf}\left(x, y, x_{s}, y_{s}\right)\right]
\end{aligned}
$$

Now let $\widetilde{\chi}_{X}(x)$ be the disjunction of all $\chi_{p}(x)$ over all patterns $p$ of words $w \in \operatorname{supp}\left(P_{X}\right) \backslash \Delta^{+}$ and let $\widetilde{\vartheta}_{X}(x, y)$ be the disjunction of all $\vartheta_{w}(x, y)$ over $w \in \operatorname{supp}\left(P_{X}\right) \cap \Delta^{+}$. Let again $w=a v_{0} X_{1} v_{1} \ldots v_{s-1} X_{s} v_{s} b \in \operatorname{supp}\left(P_{X}\right) \backslash \Delta^{+}$. Similarly to [27] we define now the formula $\tilde{\chi}_{w}(x, y)$ :

$$
\begin{aligned}
& \tilde{\chi}_{w}(x, y)=\exists y \cdot \nu(x, y) \wedge \operatorname{Lab}_{a}(x) \wedge \operatorname{Lab}_{b}(y) \wedge \\
& \wedge \exists x_{1}, y_{1}, \ldots, x_{s}, y_{s} \cdot\left[\left(x<x_{1}<y_{1} \ldots<y_{s}<y\right) \wedge \psi_{v_{0}}\left(x, x_{1}\right) \wedge \ldots \wedge \psi_{v_{s}}\left(y_{s}, y\right) \wedge\right. \\
& \wedge\left(\operatorname{surf}\left(x, y, x_{1}, y_{1}\right) \wedge \ldots \wedge \operatorname{surf}\left(x, y, x_{s}, y_{s}\right) \wedge\right. \\
& \left.\wedge\left(\widetilde{\chi}_{X_{1}}\left(x_{1}\right) \vee \vartheta_{X_{1}}\left(x_{1}, y_{1}\right)\right) \wedge \ldots \wedge\left(\widetilde{\chi}_{X_{s}}\left(x_{s}\right) \vee \vartheta_{X_{s}}\left(x_{s}, y_{s}\right)\right)\right] .
\end{aligned}
$$

We show in the next paragraph that there is a bijective correspondence between the set of derivation trees $t \in \operatorname{Der}\left(G_{Y}, u\right)$ and the nested words $(u, \nu)$ satisfying the following formula

$$
\begin{aligned}
\psi_{Y}=\exists x, y \cdot \min (x) \wedge \max (y) \wedge \nu(x, y) & \wedge\left(\tilde{\chi}_{Y}(x, y) \vee \widetilde{\vartheta}_{Y}(x, y)\right) \wedge \\
& \wedge \forall z, z^{\prime} \cdot \operatorname{inchild}\left(z, z^{\prime}\right) \rightarrow \bigvee_{\substack{X \in \mathcal{X} \\
w \in \operatorname{supp}\left(P_{X}\right) \backslash \Delta^{+}}} \tilde{\chi}_{w}\left(z, z^{\prime}\right) .
\end{aligned}
$$

The formula. Given a derivation tree $t \in \operatorname{Der}\left(G_{Y}, u\right)$ we assign to it a nesting relation $\nu_{t}$ as described above. Clearly, $(1, n) \in \nu_{t}$ and either $\left(u, \nu_{t}\right) \models \widetilde{\vartheta}_{Y}[1, n]$ or $\left(w, \nu_{t}\right) \models \widetilde{\chi}_{Y}[1, n]$. Furthermore, if $1 \leq i<j \leq n$ and $\left(u, \nu_{t}\right) \models$ inchild $[i, j]$, then there is an inner node of $t$ such that the leaves of the subtree rooted at this node are exactly the leaves between the $i$ th and the $j$ th leaf of $t$. Let $(X, w)$ be the label of this inner node, then $\left(u, \nu_{t}\right) \models \tilde{\chi}_{w}[i, j]$ 
by construction and hence $\left(u, \nu_{t}\right) \models \psi$. Conversely, let $\nu$ be a nesting relation such that $(u, \nu) \models \psi$. We define a derivation tree $t_{\nu}$ inductively as follows. If $\{(1, n)\}=\nu$, then $t_{\nu}$ consists of a single inner node, the root, labeled by $(Y, u)$. In this case we must have $(u, \nu) \models \widetilde{\vartheta}_{Y}[1, n]$ and hence $t_{\nu}$ is a derivation tree. Otherwise, let $\left(i_{1}, j_{1}\right), \ldots,\left(i_{s}, j_{s}\right)$ be the sequence of surface arches of $(u, \nu \backslash\{(1, n)\})$ and let $a_{1}^{1} \ldots a_{n_{1}}^{1}|\ldots| a_{1}^{s} \ldots a_{n_{s}}^{s} \mid a_{1}^{s+1} \ldots a_{n_{s+1}}^{s+1}$ be the pattern of $(u, \nu \backslash\{(1, n)\})$. Moreover, for $1 \leq k \leq s$ let $u\left[i_{k}, j_{k}\right]$ be the subword of $u$ from the $i_{k}$ th position to the $j_{k}$ th position. Then we must have

$$
(u, \nu) \models \bigvee_{\substack{X \in \mathcal{X} \\ w \in \operatorname{supp}\left(P_{X}\right) \backslash \Delta^{+}}} \tilde{\chi}_{w}[1, n]
$$

and hence for all $1 \leq k \leq s$ we have $(u, \nu)\left[i_{k}, j_{k}\right] \models \psi_{X_{k}}$ for some $X_{k} \in \mathcal{X}$. Thus by inductions hypothesis there are $t_{k} \in \operatorname{Der}\left(G_{X_{k}}, u\left[i_{k}, j_{k}\right]\right)$. We define $t_{\nu}$ to be the tree whose root is labeled $\left(Y, a_{1}^{1} \ldots a_{n_{1}}^{1} X_{1} \ldots X_{s} a_{1}^{s+1} \ldots a_{n_{s+1}}^{s+1}\right)$ and where the trees rooted at the children of the root are as follows from left to right: $a_{1}^{1}, \ldots, a_{n_{1}}^{1}, t_{1}, \ldots, t_{s}, a_{1}^{s+1}, \ldots, a_{n_{s+1}}^{s+1}$. We conclude that $t_{\nu}$ is a derivation tree, since $(u, \nu) \models \widetilde{\chi}_{Y}[1, n]$.

Now we can give the formula $\varphi_{Y}$.

$$
\begin{aligned}
\varphi_{Y}=\psi_{Y}^{+} \wedge \forall x, y \cdot \nu(x, y) \rightarrow & \bigvee_{\substack{X \in \mathcal{X} \\
w \in \operatorname{supp}\left(P_{X}\right)}}\left(\operatorname{inchild}(x, y) \stackrel{+}{\rightarrow}\left(\widetilde{\chi}_{w}(x, y)^{+} \wedge\left(P_{X}, w\right)\right) \wedge\right. \\
& \left.\wedge \neg \operatorname{inchild}(x, y) \stackrel{+}{\rightarrow}\left(\vartheta_{w}(x, y)^{+} \wedge\left(P_{X}, w\right)\right)\right)
\end{aligned}
$$

Let $t \in \operatorname{Der}\left(G_{Y}, u\right)$ and let $\nu_{t}$ be the corresponding nesting relation. By construction $\left(\llbracket \varphi_{Y} \rrbracket,\left(u, \nu_{t}\right)\right)=\operatorname{wgt}(t)$ and thus $\llbracket \exists \nu \cdot \varphi_{Y} \rrbracket^{\text {nest }}=S_{Y}$ by Lemma 6.4 .

Let us summarize our results of this section so far.

Theorem 6.9. Let $\mathbb{K}$ be a commutative semiring and let $S: \Delta^{*} \rightarrow \mathbb{K}$ be a formal power series. Then the following are equivalent:

(1) $S$ is an algebraic formal power series.

(2) $S=\pi(R)$ for some regular $R: \mathrm{NW}(\Delta) \rightarrow \mathbb{K}$.

(3) There is a sentence $\varphi \in \operatorname{sRFO}(\mathbb{K}, \Delta, \leq, \nu)$ such that $\llbracket \exists \nu . \varphi \rrbracket^{\text {nest }}=S$.

Proof. (1) $\Rightarrow(3)$. This is Proposition 6.8.

$(3) \Rightarrow(2)$. Follows from Theorem 3.6 (a) and the definition of $\pi$.

$(2) \Rightarrow(1)$. This is Proposition 6.3.

Let $\mathbb{K}=\mathbb{N}$ and let $S:\{a\}^{+} \rightarrow \mathbb{N}$ be an algebraic series. As $S=\pi(R)$ for some regular nested word series $R$, it is not hard to see that $\left(S, a^{n}\right) \leq 2^{n^{2}} \cdot c^{n}$ for some constant $c$ and all $n \in \mathbb{N}$. Using weighted pushdown automata (cf. [26]) one can even show that $\left(S, a^{n}\right) \leq c^{n}$ for some constant $c$ and all $n \in \mathbb{N}$. Thus in item 3 of the last result we may not replace $\operatorname{sRFO}(\mathbb{K})$ by $\mathrm{FO}(\mathbb{K})$ since $\left(\llbracket \forall x . \exists y \cdot 1 \rrbracket, a^{n}\right)=n^{n}$.

Again we note that all proofs are effective and given a proper algebraic system $\left(P_{X}\right)_{X \in \mathcal{X}}$ with solution $\left(S_{X}\right)_{X \in \mathcal{X}}$ and an effectively given semiring $\mathbb{K}$, we can compute an $\operatorname{sRFO}(\mathbb{K})$ sentence $\varphi_{Y}$ for all $Y \in \mathcal{X}$ such that $S_{Y}=\llbracket \exists \nu \cdot \varphi_{Y} \rrbracket^{\text {nest }}$ and vice versa. 
6.3. Yet Another Characterization of Algebraic Formal Power Series. Even though our logical characterization of regular nested word series (Theorem 3.6) might also be obtained by structural induction, the connection between alternating texts and nested words we established enables us now to also obtain a generalization of the second main result of [27. In this paper another logical characterization of context-free languages was given where quantification over nesting relations is now replaced by quantification over treedefinable orders. In [27] a linear order $\leq$ on $[n]$ was called tree-definable if there is a binary tree $t$ with $n$ leaves which are labeled $1, \ldots, n$ in lexicographic order and whose internal nodes are labeled with $\{\swarrow, \searrow\}$ such that $i \leq j$ iff $i$ is visited before $j$ in the depth-first traversal of $t$ in which, at every node with label $\swarrow$, first the left, and at every node with label $\searrow$, first the right child is visited. We will give a slightly different definition which is easily seen to be equivalent by simply replacing $\measuredangle$ by $\bullet$ and $\searrow$ by $\circ$.

Definition 6.10. Let $n \in \mathbb{N}_{+}$and let $\leq_{1}$ be the canonical order of $[n]$. Moreover, let $\lambda:[n] \rightarrow \Delta$ be a labeling. A linear order $\leq_{2}$ of $[n]$ is tree-definable iff $\left([n], \lambda, \leq_{1}, \leq_{2}\right)$ is an alternating text.

We collect all tree-definable orders of $[n]$ in $\mathrm{TDO}_{n}$. Our aim is now to extend the above mentioned result of [27] and to show, using the connection between nested words and alternating texts, that a formal power series is an algebraic formal power series iff it can be defined by a second-order sentence over words of the form $\exists \leq_{2} . \varphi$ where $\varphi$ is a first-order formula and $\leq_{2}$ a binary relation symbol ranging over tree-definable orders. Note that like matchings, tree-definable orders are first-order definable relations [22, 30]. First, we start by defining the projection $\pi(\tau)$ of an alternating text $\tau=\left([n], \leq_{1}, \leq_{2}, \lambda\right) \in \operatorname{TXT}(\Delta)$ to be the word $\left([n], \leq_{1}, \lambda\right)$, i.e. we forget the second order. As for nested words, this projection is canonically generalized to languages $L \subseteq \operatorname{TXT}(\Delta)$ by setting $\pi(L)=\{\pi(\tau) \mid \tau \in L\}$ and to series $S: \operatorname{TXT}(\Delta) \rightarrow \mathbb{K}$ by letting

$$
\begin{aligned}
\pi(S): \Delta^{*} & \rightarrow \mathbb{K} \\
w & \mapsto \sum_{\substack{\tau \in \operatorname{TXT}(\Delta) \\
w=\pi(\tau)}}(S, \tau) .
\end{aligned}
$$

Proposition 6.11. Let $S: \operatorname{TXT}(\Delta) \rightarrow \mathbb{K}$ be regular. Then $\pi(S): \Delta^{*} \rightarrow \mathbb{K}$ is an algebraic formal power series.

Proof. Consider a WPA $\mathcal{A}=\left(\mathcal{H}, \mathcal{V}, \Omega, \mu, \mu_{\mathrm{op}}, \mu_{\mathrm{cl}}, \lambda, \gamma\right)$ such that $\|\mathcal{A}\|=S$. Let $\mathcal{X}=$ $\left(\mathcal{H}^{2} \times\{0,1\}\right) \cup\left(\mathcal{V}^{2} \times\{0,1\}\right)$. We define an algebraic system $\left(P_{X}\right)_{X \in \mathcal{X}}$ as follows: For all $h_{1}, h_{2} \in \mathcal{H}$ and $v_{1}, v_{2} \in \mathcal{V}$ we let

$$
\begin{aligned}
\left(P_{\left(h_{1}, h_{2}, 1\right)}, w\right) & =\sum_{a \in \Delta} \mu\left(h_{1}, a, h_{2}\right) \cdot a+\sum_{v, v^{\prime} \in \mathcal{V}} \sum_{s \in \Omega} \mu_{\mathrm{op}}\left(h_{1},(s, v) \cdot \mu_{\mathrm{cl}}\left(v^{\prime},\right)_{s}, h_{2}\right) \cdot\left(v, v^{\prime}, 0\right) \\
\left(P_{\left(h_{1}, h_{2}, 0\right)}, w\right) & =\sum_{h_{3} \in \mathcal{H}}\left(h_{1}, h_{3}, 1\right)\left(h_{3}, h_{2}, 1\right)+\left(h_{1}, h_{3}, 1\right)\left(h_{3}, h_{2}, 0\right) \\
\left(P_{\left(v_{1}, v_{2}, 1\right)}, w\right) & =\sum_{a \in \Delta} \mu\left(v_{1}, a, v_{2}\right) \cdot a+\sum_{h, h^{\prime} \in \mathcal{H}} \sum_{s \in \Omega} \mu_{\mathrm{op}}\left(v_{1},(s, h) \cdot \mu_{\mathrm{cl}}\left(h^{\prime},\right)_{s}, v_{2}\right) \cdot\left(h, h^{\prime}, 0\right) \\
\left(P_{\left(v_{1}, v_{2}, 0\right)}, w\right) & =\sum_{v_{3} \in \mathcal{H}}\left(v_{1}, v_{3}, 1\right)\left(v_{3}, v_{2}, 1\right)+\left(v_{1}, v_{3}, 1\right)\left(v_{3}, v_{2}, 0\right)
\end{aligned}
$$


We claim that this algebraic system has a unique quasiregular solution $\left(S_{X}\right)_{X \in \mathcal{X}}$ which consists of algebraic formal power series. Indeed, if we replace the polynomial $P_{\left(h_{1}, h_{2}, 1\right)}$ by the polynomial

$$
\sum_{a \in \Delta} \mu\left(h_{1}, a, h_{2}\right) \cdot a+\sum_{v, v^{\prime} \in \mathcal{V}} \sum_{s \in \Omega} \mu_{\mathrm{op}}\left(h_{1},\left({ }_{s}, v\right) \cdot \mu_{\mathrm{cl}}\left(v^{\prime},\right)_{s}, h_{2}\right) \cdot P_{\left(v, v^{\prime}, 0\right)}
$$

and the polynomial $P_{\left(v_{1}, v_{2}, 1\right)}$ by the polynomial

$$
\sum_{a \in \Delta} \mu\left(v_{1}, a, v_{2}\right) \cdot a+\sum_{h, h^{\prime} \in \mathcal{H}} \sum_{s \in \Omega} \mu_{\mathrm{op}}\left(v_{1},\left({ }_{s}, h\right) \cdot \mu_{\mathrm{cl}}\left(h^{\prime},\right)_{s}, v_{2}\right) \cdot P_{\left(h, h^{\prime}, 0\right)},
$$

we obtain an equivalent system (cf. manipulations after Definition 6.2) which is proper and has thus the unique quasiregular solution $\left(S_{X}\right)_{X \in \mathcal{X}}$ which consists of algebraic formal power series. Let $\operatorname{TXT}^{\circ} \subseteq \operatorname{TXT}(\Delta)$ be the set of all alternating texts which are either singletons or o-products. Analogously let $\operatorname{TXT}^{\bullet} \subseteq \operatorname{TXT}(\Delta)$ be the set of all alternating texts which are either singletons or $\bullet$-products. We will show by induction that we have for all $w \in \Delta^{*}$ with $|w| \geq 1$

$$
\begin{aligned}
& \left(S_{\left(h_{1}, h_{2}, 1\right)}, w\right)=\sum_{\substack{\tau \in \mathrm{TXT}^{\bullet} \\
\pi(\tau)=w}} \sum_{r: h_{1} \tau} \operatorname{wgt}_{\mathcal{A}}(r) \text { and } \\
& \left(S_{\left(v_{1}, v_{2}, 1\right)}, w\right)=\sum_{\substack{\tau \in \mathrm{TXT}^{\circ} \\
\pi(\tau)=w}} \sum_{r: v_{1} \rightarrow v_{2}} \operatorname{wgt}_{\mathcal{A}}(r)
\end{aligned}
$$

as well as

$$
\begin{aligned}
\left(S_{\left(h_{1}, h_{2}, 1\right)}, w\right)+\left(S_{\left(h_{1}, h_{2}, 0\right)}, w\right) & =\sum_{\substack{\tau \in \operatorname{TXT}(\Delta) \\
\pi(\tau)=w}} \sum_{r: h_{1} \tau_{\rightarrow} \rightarrow h_{2}} \operatorname{wgt}_{\mathcal{A}}(r) \text { and } \\
\left(S_{\left(v_{1}, v_{2}, 1\right)}, w\right)+\left(S_{\left(v_{1}, v_{2}, 0\right)}, w\right) & =\sum_{\substack{\tau \in \mathrm{TXT}(\Delta) \\
\pi(\tau)=w}} \sum_{r: v_{1} \stackrel{\tau}{\rightarrow} v_{2}} \operatorname{wgt}_{\mathcal{A}}(r) .
\end{aligned}
$$

The result then follows immediately from the fact that algebraic formal power series are closed under pointwise sum and scalar multiplication. Let $w=a$ for some $a \in \Delta$. Since the series $S_{\left(h_{1}, h_{2}, 1\right)}$ and $S_{\left(v_{1}, v_{2}, 1\right)}$ are quasiregular, we obtain that $\left(S_{\left(v_{1}, v_{2}, 0\right)}, a\right)=\left(S_{\left(h_{1}, h_{2}, 0\right)}, a\right)=$ 0 . From this it is easy to deduce the induction base. Let now $|w|>1$. Then

$$
\begin{aligned}
& \left(S_{\left(h_{1}, h_{2}, 1\right)}, w\right)=\sum_{v, v^{\prime} \in \mathcal{V}} \sum_{s \in \Omega} \mu_{\mathrm{op}}\left(h_{1},(s, v) \cdot \mu_{\mathrm{cl}}\left(v^{\prime},\right)_{s}, h_{2}\right) \cdot\left(S_{\left(v, v^{\prime}, 0\right)}, w\right) \\
& =\sum_{v, v^{\prime} \in \mathcal{V}} \sum_{s \in \Omega} \mu_{\mathrm{op}}\left(h_{1},(s, v)\right. \\
& \left.\quad\left(\sum_{v_{3} \in \mathcal{V}} \sum_{w=w_{1} w_{2}}\left(S_{\left(v, v_{3}, 1\right)}, w_{1}\right) \cdot\left(S_{\left(v_{3}, v^{\prime}, 1\right)}, w_{2}\right)+\left(S_{\left(v, v_{3}, 1\right)}, w_{1}\right) \cdot\left(S_{\left(v_{3}, v^{\prime}, 0\right)}, w_{2}\right)\right) \cdot \mu_{\mathrm{cl}}\left(v^{\prime},\right)_{s}, h_{2}\right) \\
& =\sum_{v, v^{\prime} \in \mathcal{V}} \sum_{s \in \Omega} \mu_{\mathrm{op}}\left(h_{1},(s, v) \cdot\right. \\
& \left.\quad\left(\sum_{v_{3} \in \mathcal{V}} \sum_{w=w_{1} w_{2}}\left(S_{\left(v, v_{3}, 1\right)}, w_{1}\right) \cdot\left(\left(S_{\left(v_{3}, v^{\prime}, 1\right)}, w_{2}\right)+\left(S_{\left(v_{3}, v^{\prime}, 0\right)}, w_{2}\right)\right)\right) \cdot \mu_{\mathrm{cl}}\left(v^{\prime},\right)_{s}, h_{2}\right)
\end{aligned}
$$




$$
\begin{aligned}
& =\sum_{v, v^{\prime} \in \mathcal{V}} \sum_{s \in \Omega} \mu_{\mathrm{op}}\left(h_{1},{ }_{s}, v\right) \text {. } \\
& \left.\left(\sum_{v_{3} \in \mathcal{V}} \sum_{w=w_{1} w_{2}} \sum_{\substack{\tau_{1} \in \mathrm{TXT}^{\circ} \\
\pi\left(\tau_{1}\right)=w_{1}}} \sum_{r_{1}: v \rightarrow \tau_{3} \rightarrow v_{3}} \operatorname{wgt}_{\mathcal{A}}\left(r_{1}\right) \cdot \sum_{\substack{\tau_{2} \in \mathrm{TXT}(\Delta) \\
\pi\left(\tau_{2}\right)=w_{2}}} \sum_{r_{1}: v_{3} \tau_{2} \rightarrow v^{\prime}} \operatorname{wgt}_{\mathcal{A}}\left(r_{1}\right)\right) \cdot \mu_{\mathrm{cl}}\left(v^{\prime},\right)_{s}, h_{2}\right)
\end{aligned}
$$

Since $\operatorname{TXT}(\Delta)$ is the free bisemigroup, given some $w$ of length at least two, each $\tau \in \mathrm{TXT}^{\bullet}$ with $\pi(\tau)=w$ decomposes uniquely into $\tau=\tau_{1} \bullet \tau_{2}$ with $\tau_{1} \in \mathrm{TXT}^{\circ}$ and $\tau_{2} \in \operatorname{TXT}(\Delta)$. Hence we can continue

$$
=\sum_{\substack{\tau \in \mathrm{TXT}^{\bullet} \\ \pi(\tau)=w}} \sum_{r: h_{1}{ }^{\tau} \rightarrow h_{2}} \operatorname{wgt}_{\mathcal{A}}(r) .
$$

Analogously we get Equation (6.4). Similarly we get:

$$
\begin{aligned}
& \left(S_{\left(h_{1}, h_{2}, 1\right)}, w\right)+\left(S_{\left(h_{1}, h_{2}, 0\right)}, w\right)= \\
& =\sum_{\substack{\tau \in \mathrm{TXT}^{\bullet} \\
\pi(\tau)=w}} \sum_{r: h_{1} \rightarrow h_{2}} \operatorname{wgt}_{\mathcal{A}}(r)+\sum_{h_{3} \in \mathcal{H}} \sum_{w=w_{1} w_{2}}\left(S_{\left(h_{1}, h_{3}, 1\right)}, w_{1}\right) \cdot\left(\left(S_{\left(h_{3}, h_{2}, 1\right)}, w_{2}\right)+\left(S_{\left(h_{3}, h_{2}, 0\right)}, w_{2}\right)\right) \\
& =\sum_{\substack{\tau \in \mathrm{TXT}^{\bullet} \\
\pi(\tau)=w}} \sum_{r: h_{1} \tau_{\rightarrow} h_{2}} \operatorname{wgt}_{\mathcal{A}}(r)+
\end{aligned}
$$

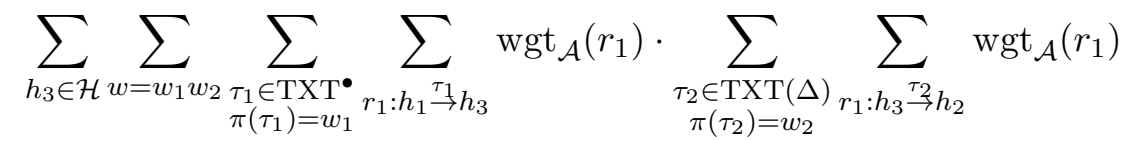

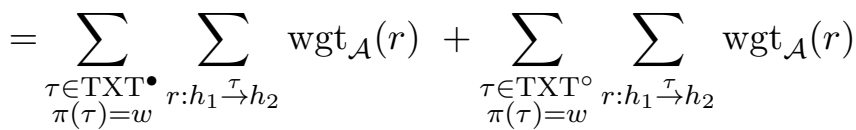

$$
\begin{aligned}
& =\sum_{\substack{\tau \in \operatorname{TXT}(\Delta) \\
\pi(\tau)=w}} \sum_{r: h_{1} \stackrel{\tau}{\rightarrow} h_{2}} \operatorname{wgt}_{\mathcal{A}}(r) .
\end{aligned}
$$

Again Equation (6.6) can be shown analogously, which concludes the proof.

Now we get our second characterization of algebraic formal power series. For this, we proceed as follows: Let $\varphi$ be a weighted second-order formula over words containing, apart from a single 2 -ary relation variable $\leq_{2}$, only 1-ary relation variables. In other words, let $\varphi \in \operatorname{MSO}\left(\mathbb{K}, \Delta, \leq_{1}, \leq_{2}\right)$. Let $\operatorname{Free}(\varphi) \subseteq \mathcal{V}, w=\left([n], \leq_{1}, \lambda\right) \in \Delta^{*}$ and $\gamma$ a $(\mathcal{V}, w)$-assignment. We define the semantics $\llbracket \exists \leq_{2} \cdot \varphi \rrbracket^{\text {tdo }}: \Delta^{*} \rightarrow \mathbb{K}$ by letting

$$
\left(\llbracket \exists \leq_{2} \cdot \varphi \rrbracket^{\mathrm{tdo}},(w, \gamma)\right)=\sum_{\leq_{2} \in \mathrm{TDO}_{n}}\left(\llbracket \varphi \rrbracket,\left(\left([n], \leq_{1}, \leq_{2}, \lambda\right), \gamma\right)\right) .
$$

Theorem 6.12. Let $\mathbb{K}$ be a commutative semiring and let $S: \Delta^{*} \rightarrow \mathbb{K}$ be a formal power series. Then the following are equivalent:

(1) $S$ is an algebraic formal power series.

(2) $S=\pi(R)$ for some regular $R: \operatorname{TXT}(\Delta) \rightarrow \mathbb{K}$.

(3) There is a sentence $\varphi \in \operatorname{sRFO}\left(\mathbb{K}, \Delta, \leq_{1}, \leq_{2}\right)$ such that $\llbracket \exists \leq_{2} \cdot \varphi \rrbracket^{t d o}=S$. 
Proof. (1) $\Rightarrow(3)$. Let $S: \Delta^{*} \rightarrow \mathbb{K}$ be an algebraic formal power series. By Theorem 6.9 there is an $\operatorname{sRFO}(\mathbb{K})$ sentence over nested words such that $\llbracket \exists \nu . \varphi \rrbracket^{\text {nest }}=S$. By Corollary $[5.6$ the partial function $\Phi_{\circ}^{-1}$ is FO-definable without parameter. Similar to Proposition 5.2 one can show that there is thus an $\operatorname{sRFO}(\mathbb{K})$ sentence $\varphi^{\prime}$ over texts such that $\Phi_{\circ}(\llbracket \varphi \rrbracket)=\llbracket \varphi^{\prime} \rrbracket$. Now we can calculate using observations (国) and (b) before Lemma 5.3 as follows.

$$
\begin{aligned}
\left(\llbracket \exists \nu \cdot \varphi \rrbracket^{\text {nest }}, w\right) & =\sum_{\substack{n w \in \mathrm{NW}(\Delta) \\
\pi(n w)=w}}(\llbracket \varphi \rrbracket, n w)=\sum_{\substack{n w \in \mathrm{NW}(\Delta) \\
\pi\left(\Phi_{\circ}(n w)\right)=w}}(\llbracket \varphi \rrbracket, n w) \\
= & \sum_{\substack{\tau \in \Phi_{\circ}(\mathrm{NW}(\Delta)) \\
\pi(\tau)=w}}\left(\llbracket \varphi \rrbracket, \Phi_{\circ}^{-1}(\tau)\right)=\sum_{\substack{\tau \in \Phi_{\circ}(\mathrm{NW}(\Delta)) \\
\pi(\tau)=w}}\left(\llbracket \varphi^{\prime} \rrbracket, \tau\right)=\left(\llbracket \exists \leq_{2} \cdot \varphi^{\prime} \rrbracket^{\text {tdo }}, w\right) .
\end{aligned}
$$

$(3) \Rightarrow(2)$. Follows from Theorem 4.2 and the definition of $\pi$.

$(2) \Rightarrow(1)$. This is Proposition 6.11.

\section{Concluding Remarks and Future Work}

We introduced a quantitative automaton model and a quantitative logic for nested words and showed that they are equally expressive. This generalizes the logical characterization of the unweighted case as given in [4]. Moreover, we established a new connection between nested words and alternating texts. Applying the result, we obtained a characterization of algebraic formal power series in terms of weighted logics. Presumably, the logical characterization of regular nested word series could also be obtained by structural induction. However, the connection between alternating texts and nested words enabled us to also obtain a second characterization of algebraic formal power series. Note that even though the characterizations of algebraic formal power series are generalizations of the results of [27] to a weighted setting, in contrast to the latter paper we gave a different proof using this connection as well as (weighted) nested word automata and (weighted) parenthesizing automata. Also note that weighted nested word automata and weighted parenthesizing automata were characterized algebraically in 31.

Let us remark that regular formal power series also fall into the pattern of our characterizations (Theorem 6.9 and Theorem 6.12) of algebraic formal power series. In fact, Thomas showed that a single existential monadic second-order quantifier suffices to characterize finite automata [39, Theorem 5.2]. That is, in the pattern of the last results we can formulate that $L \subseteq \Delta^{*}$ is regular iff $L=\llbracket \exists M . \varphi \rrbracket^{\text {set }}$ for some first-order formula $\varphi$ (where $\llbracket \exists M . \varphi \rrbracket^{\text {set }}$ means that we sum over all subsets $M$ of the domain of a given structure). Let us explain the idea of the proof with an example. Given an automaton $\mathcal{A}$ with set of states $Q=\{0,1\}^{k}$ for $L$ and some word $a_{1} \ldots a_{2 k} \in L$, the idea is to think of the interpretation of $M$ as a word $u_{1} u_{2}$ where $u_{1}, u_{2} \in\{0,1\}^{k}=Q$ and to express by $\varphi$ that $u_{1}$ is an initial state, that there is a run from $u_{1}$ to $u_{2}$ on $a_{1} \ldots a_{k}$ and that there is a run from $u_{2}$ into a final state on $a_{k+1} \ldots a_{2 k}$. Alternatively, one can prove the result similarly to Proposition 6.8 where one starts with a right-regular system and applies a similar transformation. Then a set $M$ suffices to encode a derivation tree since any inner node has at most one non-terminal child whose position is collected in $M$. In any way, it is not hard to see that the proof can be adapted to a weighted setting. So, also in the weighted case we can restrict ourselves to a single existential monadic second-order quantifier. 
Following these pattern it might be interesting to further investigate whether other important classes of formal power series can be characterized in this manner. Again, the work of Lautemann, Schwentick and Thérien [27] can be used as a starting point where the so-called $k$-linear languages were considered.

\section{ACKNOWLEDGMENTS.}

The author thanks Manfred Droste and Andreas Maletti for helpful comments, Dietrich Kuske for pointing him to [27]. Moreover, he thanks the anonymous referees of this journal version and the referees of the conference version. Their careful reading and their remarks resulted in substantial improvements.

\section{REFERENCES}

[1] R. Alur, M. Arenas, P. Barceló, K. Etessami, N. Immerman, and L. Libkin. First-order and temporal logics for nested words. Logical Methods in Computer Science, 4(4:11):1-44, 2008.

[2] R. Alur, V. Kumar, P. Madhusudan, and M. Viswanathan. Congruences for visibly pushdown languages. In Proc. of the 32nd ICALP, Lisbon, volume 3580 of Lecture Notes in Computer Science, pages 11021114, 2005.

[3] R. Alur and P. Madhusudan. Visibly pushdown languages. In Proc. of the 36th STOC, Chicago, pages 202-211. ACM, 2004

[4] R. Alur and P. Madhusudan. Adding nesting structure to words. In Proc. of the 10th DLT, Santa Barbara, volume 4036 of Lecture Notes in Computer Science, pages 1-13, 2006.

[5] C. Baier, F. Ciesinski, and M. Größer. Model checking linear-time properties of probabilistic systems. In Droste et al. [13], chapter 13.

[6] C. Baier and M. Größer. Recognizing omega-regular languages with probabilistic automata. In Proc. of the 20th LICS, Chicago, pages 137-146. IEEE Computer Society, 2005.

[7] P. Chervet and I. Walukiewicz. Minimizing variants of visibly pushdown automata. In Proc. of the 32nd MFCS, Ceský Krumlov, volume 4708 of Lecture Notes in Computer Science, pages 135-146, 2007.

[8] N. Chomsky and M.P. Schützenberger. The algebraic theory of context-free languages. In Computer Programming and Formal Systems, pages 118-161. North-Holland Publishing Company, 1963.

[9] B. Courcelle. Monadic second-order definable graph transductions: a survey. Theoretical Computer Science, 126:53-75, 1994.

[10] K. Culik II and J. Kari. Image compression using weighted finite automata. Computer Es Graphics, 17(3):305 - 313, 1993.

[11] M. Droste and P. Gastin. Weighted automata and weighted logics. Theoretical Computer Science, 380:69-86, 2007.

[12] M. Droste and P. Gastin. Weighted automata and weighted logics. In Droste et al. 13, chapter 5.

[13] M. Droste, W. Kuich, and H. Vogler, editors. Handbook of Weighted Automata. EATCS Monographs on Theoretical Computer Science. Springer, 2009.

[14] M. Droste and G. Rahonis. Weighted automata and weighted logics on infinite words. In Proc. of the 10th DLT, Santa Barbara, volume 4036 of Lecture Notes in Computer Science, pages 49-58, 2006.

[15] M. Droste and H. Vogler. Weighted tree automata and weighted logics. Theoretical Computer Science, $366: 228-247,2006$.

[16] A. Ehrenfeucht and G. Rozenberg. T-structures, T-functions, and texts. Theoretical Computer Science, 116:227-290, 1993.

[17] Z. Ésik and Z.L. Németh. Higher dimensional automata. Journal of Automata, Languages and Combinatorics, 9(1):3-29, 2004.

[18] I. Fichtner. Weighted picture automata and weighted logics. Theory of Computing Systems, 2009. in press; ; extended abstract appeared as 32 .

[19] K. Hashiguchi, S. Ichihara, and S. Jimbo. Formal languages over free binoids. Journal of Automata, Languages and Combinatorics, 5(3):219-234, 2000. 
[20] K. Hashiguchi, S. Jimbo, and T. Kunai. Finite codes over free binoids. Journal of Automata, Languages and Combinatorics, 7(4):505-518, 2002.

[21] K. Hashiguchi, S. Jimbo, and Y. Wada. Regular binoid expressions and regular binoid languages. Theoretical Computer Science, 1-3(304):291-313, 2003.

[22] H.J. Hoogeboom and P. ten Pas. Monadic second-order definable text languages. Theory of Computing Systems, 30:335-354, 1997.

[23] S. Jha, D. Melski, T.W. Reps, and S. Schwoon. Weighted pushdown systems and their application to interprocedural dataflow analysis. Science of Computer Programming, 58(1-2):206-263, 2005.

[24] N. Kidd, A. Lal, and T.W. Reps. Program analysis using weighted pushdown systems. In Proc. of the 27th FSTTCS 2007, New Delhi, volume 4855 of Lecture Notes in Computer Science, pages 23-51, 2007.

[25] W. Kuich. Semirings and formal power series. In G. Rozenberg and A. Salomaa, editors, Word, Language, Grammar, volume 1 of Handbook of Formal Languages, chapter 9, pages 609-677. Springer, 1997.

[26] W. Kuich and A. Salomaa. Semirings, Automata, Languages, volume 5 of EATCS Monographs on Theoretical Computer Science. Springer, 1986.

[27] C. Lautemann, T. Schwentick, and D. Thérien. Logics for context-free languages. In Proc. of the 8th CSL, Kazimierz, volume 933 of Lecture Notes in Computer Science, pages 205-216, 1994.

[28] C. Mathissen. Definable transductions and weighted logics for texts. In Proc. of the 11th DLT, Turku, volume 4588 of Lecture Notes in Computer Science, pages 324-336, 2007.

[29] C. Mathissen. Weighted logics for nested words and algebraic formal power series. In Proc. of the 35th ICALP, Reykjavik, Part II, volume 5126 of Lecture Notes in Computer Science, pages 221-232, 2008.

[30] C. Mathissen. Definable transductions and weighted logics for texts. Theoretical Computer Science, 2009. in press; extended abstract appeared as 28.

[31] C. Mathissen. Weighted Automata and Weighted Logics over Tree-like Structures. PhD Thesis, Universität Leipzig, 2009.

[32] I. Mäurer. Weighted picture automata and weighted logics. In Proc. of the 23rd STACS, Marseille, volume 3884 of Lecture Notes in Computer Science, pages 313-324, 2006.

[33] I. Meinecke. Weighted logics for traces. In Proc. of the 1st CSR, St. Petersburg, volume 3967 of Lecture Notes in Computer Science, pages 235-246, 2006.

[34] M. Mohri. Finite-state transducers in language and speech processing. Computational Linguistics, 23:269-311, 1997.

[35] I. Petre and A. Salomaa. Algebraic systems and pushdown automata. In Droste et al. [13, chapter 7.

[36] G. Rahonis. Weighted muller tree automata and weighted logics. Journal of Automata, Languages and Combinatorics, 12(4):455-483, 2007.

[37] A. Salomaa and M. Soittola. Automata-Theoretic Aspects of Formal Power Series. Texts and Monographs in Computer Science. Springer, 1978.

[38] S. Schwarz. Łukasiewicz logics and weighted logics over MV-semirings. Journal of Automata, Languages and Combinatorics, 12(4):485-499, 2007.

[39] W. Thomas. Classifying regular events in symbolic logic. Journal of Computer and System Sciences, $25(3): 360-376,1982$. 